\title{
Evaluation of Induced Settlements of Piled Rafts in the Coupled Static-Dynamic Loads Using Neural Networks and Evolutionary Polynomial Regression
}

\author{
Ali Ghorbani and Mostafa Firouzi Niavol \\ Department of Civil Engineering, Faculty of Engineering, The University of Guilan, Rasht, Iran \\ Correspondence should be addressed to Ali Ghorbani; ghorbani@guilan.ac.ir
}

Received 30 January 2017; Revised 27 April 2017; Accepted 6 June 2017; Published 19 July 2017

Academic Editor: Sandeep Chaudhary

Copyright ( 2017 Ali Ghorbani and Mostafa Firouzi Niavol. This is an open access article distributed under the Creative Commons Attribution License, which permits unrestricted use, distribution, and reproduction in any medium, provided the original work is properly cited.

\begin{abstract}
Coupled Piled Raft Foundations (CPRFs) are broadly applied to share heavy loads of superstructures between piles and rafts and reduce total and differential settlements. Settlements induced by static/coupled static-dynamic loads are one of the main concerns of engineers in designing CPRFs. Evaluation of induced settlements of CPRFs has been commonly carried out using threedimensional finite element/finite difference modeling or through expensive real-scale/prototype model tests. Since the analyses, especially in the case of coupled static-dynamic loads, are not simply conducted, this paper presents two practical methods to gain the values of settlement. First, different nonlinear finite difference models under different static and coupled static-dynamic loads are developed to calculate exerted settlements. Analyses are performed with respect to different axial loads and pile's configurations, numbers, lengths, diameters, and spacing for both loading cases. Based on the results of well-validated three-dimensional finite difference modeling, artificial neural networks and evolutionary polynomial regressions are then applied and introduced as capable methods to accurately present both static and coupled static-dynamic settlements. Also, using a sensitivity analysis based on Cosine Amplitude Method, axial load is introduced as the most influential parameter, while the ratio 1/d is reported as the least effective parameter on the settlements of CPRFs.
\end{abstract}

\section{Introduction}

CPRF is representative for combined piled raft foundation, which is commonly applied to suffer the heavy load of skyscrapers through sharing the exerted load between raft and piles. Coefficient of piled raft, $\alpha_{\mathrm{pr}}$, controls load sharing ratio and is defined as the ratio of the sum of the loads carried by piles to the corresponding value of the resistance of the whole system. It varies from 0 for spread footings to 1 for pile foundations. Also, as shown in Figure 1, the settlements of a CPRF $\left(S_{\mathrm{pr}}\right)$ can be calculated based on the value of $\left(\alpha_{\mathrm{pr}}\right)$ and the settlement of pile foundation $\left(S_{\mathrm{pf}}\right)$.

Piles are generally used to decrease the foundation's total settlements. In the last three decades, some researchers like those of [1-3] have declared efficient load sharing mechanisms to accurately investigate the piled raft behavior.
New well-developed computational approaches and available finite element (FE) codes such as Defpig and Napra have facilitated modeling of available interactions [4-6]. Besides, some other small scale model studies $[7,8]$ and numerical and analytical simulations in the literature have made improvements in the design process [9-11]. In addition, results of monitoring the behavior of piled rafts supporting heavy loads of some skyscrapers are reported $[12,13]$. But there is an obvious need for a comprehensive study about the piled raft settlements in the case of combined vertical static loads and horizontal dynamic ones. Since it is experimentally time-consuming and expensive, numerical modeling is considered as a good alternative to accurately study CPRFs behavior. However, in order to have a comprehensive and accurate model, especially in the case of CPRFs for high rise buildings with large number of piles, there will be a large number of structural elements 


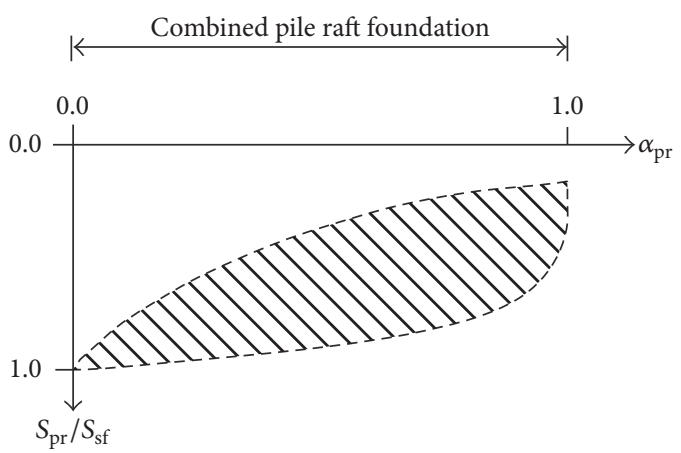

FIGURE 1: Settlement of a CPRF.

and the calculation time makes the analysis so difficult. To cope with this difficulty, recently, some research works are focused on the application of soft computing techniques, as the speedy and powerful tool and alternative for other timeconsuming and expensive methods, to the problem of piles and piled raft foundations behaviors $[14,15]$. In the research of Armaghani et al. (2015) [14], the ultimate bearing capacity is investigated, while Baziar et al. (2014) [15] evaluated the static induced settlements. Hence, the dynamically loaded induced settlements of piled rafts and the application of soft computing techniques to this problem have not been completely studied. Then, there is an obvious need for a speedy and accurate tool for predicting piled raft settlements in the combined vertical static-horizontal dynamic loading conditions. Relying on the fact that conducting real-scale or prototype scale field or experimental tests on the dynamic behavior of Coupled Piled Raft Foundations needs a lot of time and money and also considering the process and analysis time of numerical modeling, if an alternative method for predicting the settlements of CPRFs in dynamic loading cases exists, it will be useful. This method should have advantages of both accurate experimental modeling and low calculation time. Hence, the application of neural network modeling and evolutionary polynomial regressions (EPRs), which are believed to be common ways to accurately and timely predict engineering complicated functions, can be examined. Different attempts to apply neural networks and EPRs to model different civil and geotechnical problems are presented in the literature [14-33]. They are well-applied in a wide range of problems from deep soil stabilizations, concrete, and their related structures, compressive strength of soils, rocks, and stabilized samples, bearing capacity of shallow and deep foundations, lateral spreading, rock mechanics, rock engineering, and soil mechanics [14-33]. In these methods, a sufficient amount of accurate data is required initially to train artificial neural networks and evolutionary polynomial regression models. In this regard, firstly, based on nonlinear dynamic finite difference method, piled raft foundation models were developed and successfully validated with available field tests. Based on the parametric study on some geometrical parameters of CPRF and loads, a comprehensive sight to the problem has been achieved and a strong database has been prepared and results of induced settlements for both static and coupled static-dynamic conditions are calculated and gathered regarding input concerning parameters. Then, using new powerful forecasting methods, for example, neural network modeling and evolutionary polynomial regression modeling, it has been attempted to present comprehensive, speedy, and accurate models for predicting CPRF's settlements in static and combined staticdynamic loading conditions. Hence, as the novelty of the paper, new neural network and EPR models are presented for modeling settlements of CPRF in the static and coupled static-dynamic loading conditions. As another main result of the paper, the most and the least influential parameters, which, respectively, should be paid more and less attentions in dynamic CPRF modeling, are introduced.

\section{Nonlinear Finite Difference Modeling of CPRF}

In order to develop CPRF models, FLAC 3D software was used and CPRFs with regular $3 \times 3,4 \times 4$, and $5 \times 5$ arrangements were modeled in both static and combined static-dynamic conditions.

In order to achieve a comprehensive model, three different arrangements and topologies were considered for piles (pattern $\mathrm{N}$ for near piles, pattern $\mathrm{M}$ for mediate piles, and pattern $\mathrm{F}$ for far piles). Also, 4, 5, and 6 were the ratios of $S / D$ (spacing of piles to the piles' diameters). Also, assuming four $(l / D)$ conditions $(16,20,24$, and 32) and two different static vertical pressure states $(60$ and $90 \mathrm{kPa}$ ) static and combined static-dynamic settlements were calculated.

In order to model three-dimensional soil and pile geometry, 8-node brick elements and 6-node cylinder elements are, respectively, used. Names, shapes and other specifications of the used mesh shapes are presented in Figure 2.

Also, there are some well-developed constitutive models presented in FLAC as built in material behaviors. Among them, Mohr-Coulomb's model applies more simple constitutive parameters (in view of characterization), internal friction angle, cohesion, and dilation. Hence, it is considerably applied to model the behavior of surrounding soil. Also, in order to investigate the interaction between the soil and piles, shear and normal coupling springs are used. In this paper, using nonassociative Mohr-Coulomb model (the yield surface is presented in Figure 3), the soil nonlinear behavior is modeled, while slippage and separation during the motion are described using coupling springs. The normal behavior of the pile/grid interface is represented by a spring with a limiting normal force, which is dependent on the direction of piles' nodal movement. In addition, the shear behavior of the pile/grid interface is represented as a spring-slider system at the nodes of modeled piles. The shear behavior of the interface during relative displacement between the nodal points of pile and the grid is numerically attained by adjusting the proper shear and normal stiff-nesses. Elements of the interface and parameters of the used constitutive model in the interface along with the interface elements along the bottom and the side are presented in Figure 4. 


\begin{tabular}{|c|c|c|c|c|c|c|}
\hline Shape & Name & Keyword & $\begin{array}{l}\text { Reference } \\
\text { points }\end{array}$ & $\begin{array}{c}\text { Size } \\
\text { entries }\end{array}$ & $\begin{array}{c}\text { Dimension } \\
\text { entries }\end{array}$ & Fill \\
\hline & Brick & Brick & 8 & 3 & 0 & No \\
\hline & $\begin{array}{l}\text { Degenerate } \\
\text { brick }\end{array}$ & Dbrick & 7 & 3 & 0 & No \\
\hline & Wedge & Wedge & 6 & 3 & 0 & No \\
\hline & $\begin{array}{l}\text { Uniform } \\
\text { wedge }\end{array}$ & Uwedge & 6 & 3 & 0 & No \\
\hline & Pyramid & Pyramid & 5 & 3 & 0 & No \\
\hline & Tetrahedron & Tetrahedron & 4 & 3 & 0 & No \\
\hline & Cylinder & Cylinder & 6 & 3 & 0 & No \\
\hline
\end{tabular}

Figure 2: Specifications of the used elements [16].

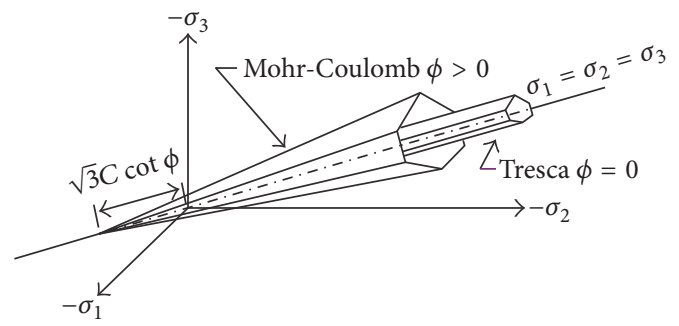

Figure 3: Mohr-Coulomb material model used to investigate the nonlinear soil behavior [6].

For the analysis of the piled raft foundation, the soil as well as the structure is discretized into elements. Usual practice is to divide the soil mass into rectangular zones of aspect ratios less than $4: 1$. For the region of greater interest, finer discretization is used. The software internally breaks each of the rectangular elements into four overlapping triangular elements. The limits of the discretized grid should be properly planned to suit the geometry of the problem. To avoid boundary effects, the mesh should extend sufficiently beyond the region of interest. Experiences of the previous researchers are taken as guidelines to decide the extent of the grid. The software also allows graded discretization, which is more efficient than abrupt change in zone sizes. In this regard, a sensitivity analysis on the number of grids (mesh refinement) is conducted to obtain the proper mesh size, in which the size of elements does not meaningfully change the accuracy of results. Figure 5 presents the adopted mesh for piles cap and the soil and the pile's length in the optimum refined mesh view. A typical shape for one of the developed models has been shown in Figure 6, where elements near the piles are finer than others to accurately track the sensitivities in this zone (with higher stress intensity). In addition, in order to neglect the boundary effects, the lateral boundaries are in the distances equal to $50 \mathrm{D}$ from the edge of raft. Moreover, the distance between lower boundaries with the tip of piles is assumed to be $3 L$ (length of piles). It should be noted that interactions between soil and piles have been accurately considered using interface elements and [34]

$$
k_{s}=k_{n}=10 \max \left(\frac{K+4 / 3 G}{\Delta z_{\min }}\right) \text {, }
$$

where $K$ is the soil's bulk modulus, $G$ is the shear modulus of the soil, $\Delta z_{\min }$ is the minimum width of neighbor elements at the interface, and $k_{s}$ and $k_{n}$, respectively, present shear and normal stiffness of elements at interface nodes.

2.1. Dynamic Analysis. Critical time step is calculated using [34]

$$
\Delta t_{\text {crit }}=\min \left\{\frac{A}{C_{P} \Delta x_{\max }}\right\},
$$

where $C_{p}$ is the speed of $P$ wave, $A$ represents the area of triangular element, and $\Delta x_{\max }$ is the maximum dimension of the area. In the case of damping proportional to the stiffness, the critical time step is calculated using [36]

$$
\Delta t_{\beta}=\left\{\frac{2}{\omega_{\max }}\right\}\left(\sqrt{1+\lambda^{2}}-\lambda\right),
$$




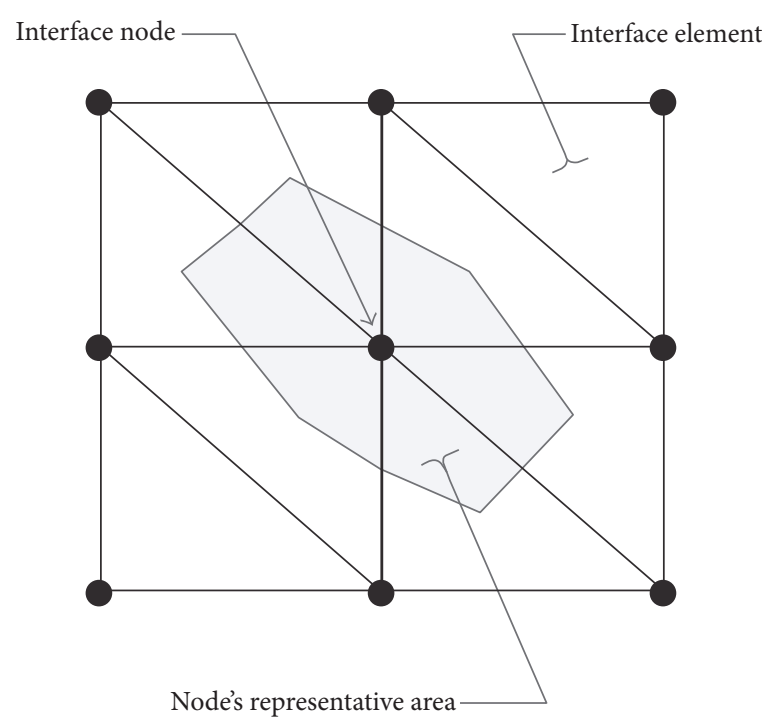

(a)

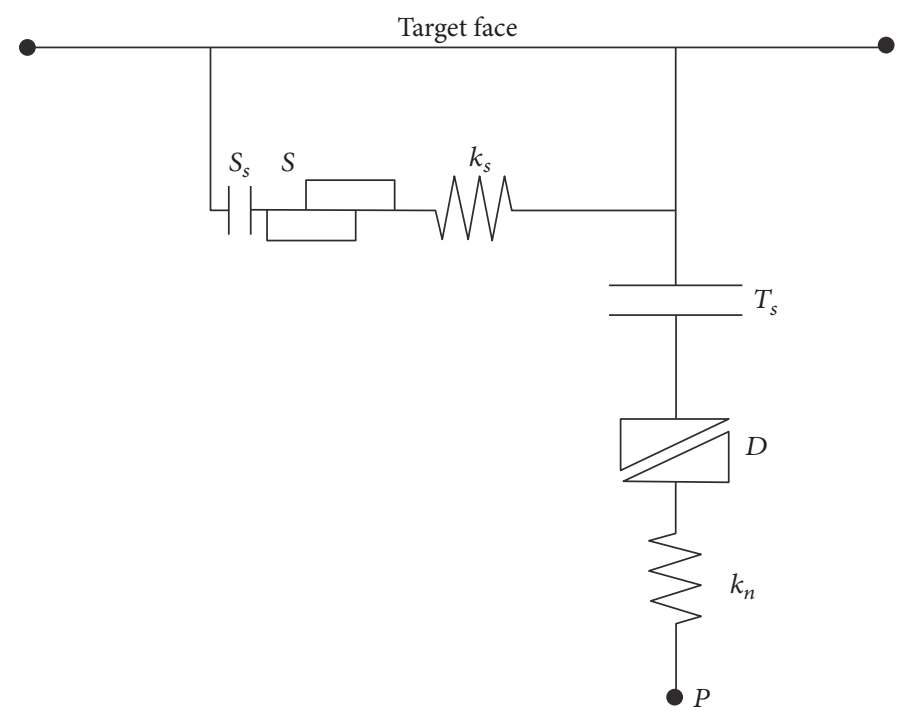
$S=$ Slider
$T_{s}=$ Tensile strength

$$
\begin{aligned}
& D=\text { Dilation } \\
& k_{s}=\text { Shear stiffness }
\end{aligned}
$$

$S_{s}=$ Shear strength$$
k_{n}=\text { Normal stiffness }
$$

(b)

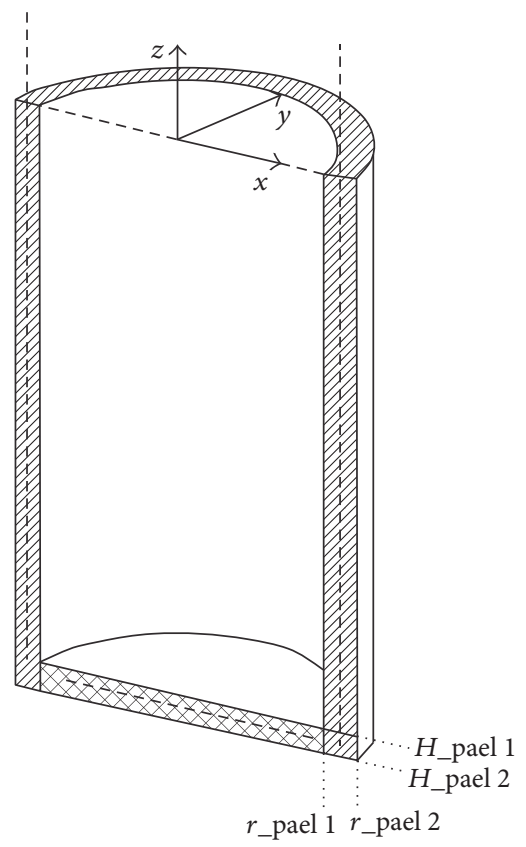

留 Interface along sides

Interface at bottom

(c)

FIGURE 4: (a) Interface elements; (b) constitutive parameters in the interface; (c) bottom and side interface elements [16].

where $\omega_{\max }$ is maximum predominant frequency of system and $\lambda$ represents a ratio of critical damping at this frequency and are calculated using [34]

$$
\omega_{\max }=\frac{2}{\Delta t_{d}}
$$

$$
\begin{aligned}
& \lambda=\frac{0.4 \beta}{\Delta t_{d}}, \\
& \beta=\frac{\xi_{\text {min }}}{\omega_{\text {min }}} .
\end{aligned}
$$



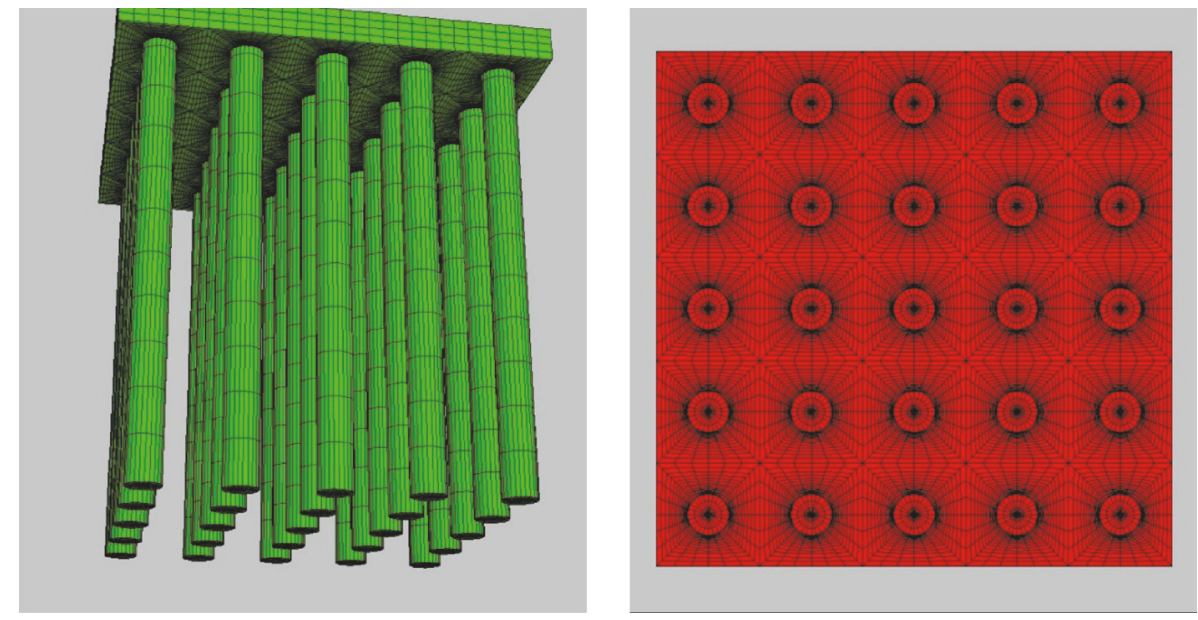

FIGURE 5: Two different grid sizes used to carry out the sensitivity analysis on the mesh sizes.
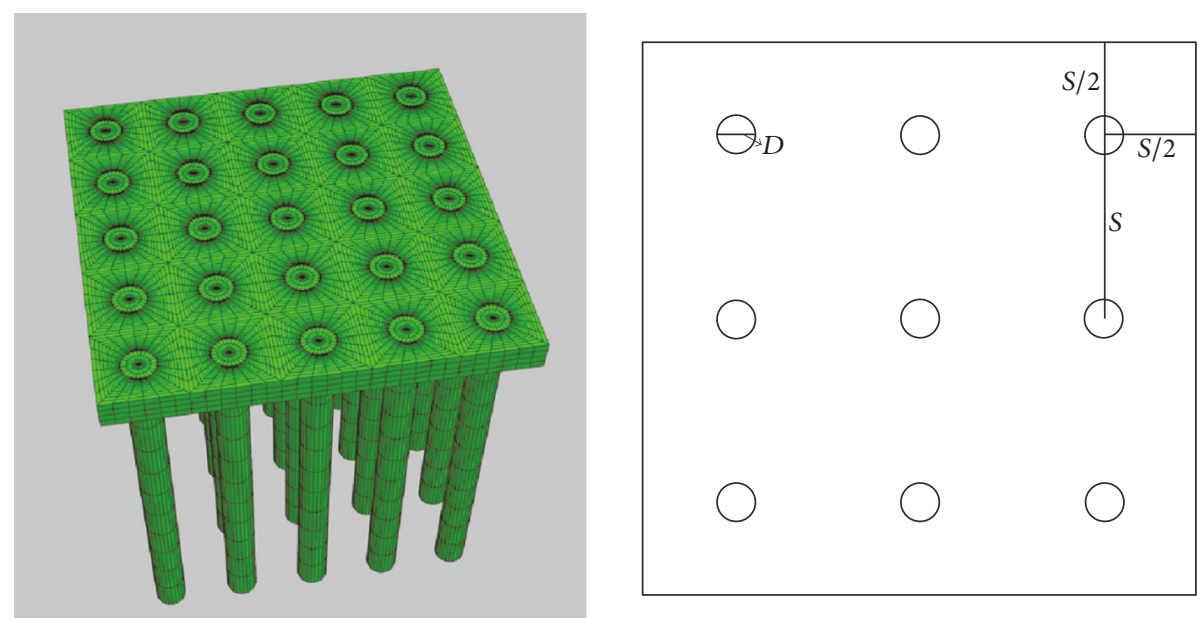

FIGURE 6: Developed three-dimensional FD model.

In these equations, $\xi_{\min }$ and $\omega_{\min }$ are Rayleigh's damping and angular frequency.

2.2. Boundary Conditions. Quiet boundaries introduced by Lysmer and Kuhlemeyer [34] are used to neglect the effects of wave reflections in the model. In these boundaries, normal and shear quiet tensions are modeled using [34]

$$
\begin{aligned}
& t_{n}=-\rho C_{P} v_{n}, \\
& t_{S}=-\rho C_{S} v_{S},
\end{aligned}
$$

where $v_{n}$ and $v_{S}$ are, respectively, normal and shear components of wave velocity at boundary. $\rho$ is specific gravity and $C_{P}$ and $C_{S}$ represent $P$ and $S$ wave's speed, respectively. Also, $t_{n}$ and $t_{S}$, respectively, show the normal and shear quiet tensions. Figure 7 shows quiet boundaries used in FLAC for rigid and flexible beds.
2.3. Loading Condition. As mentioned, in order to evaluate the effect of coupled vertical static loading and horizontal dynamic loading, 60 and $90 \mathrm{kPa}$ vertical loads were separately exerted to the structure and the model was analyzed. Also, dynamic loading was modeled using horizontal stress wave as shown in Figure 8. Since quiet boundaries were used, acceleration/speed time histories at boundaries cannot be exerted to the models. Hence, based on (6) [34], the wave velocities were changed to the waves' stress terms and shown in Figure 8.

$$
\begin{aligned}
& \sigma_{n}=2\left(\rho C_{P}\right) v_{n}, \\
& \sigma_{S}=2\left(\rho C_{S}\right) v_{S},
\end{aligned}
$$

where $v_{n}$ and $v_{S}$ are, respectively, normal and shear components of the wave velocity at boundary. $\rho$ is specific gravity and $C_{P}$ and $C_{S}$ represent $P$ and $S$ wave's velocity, respectively. 


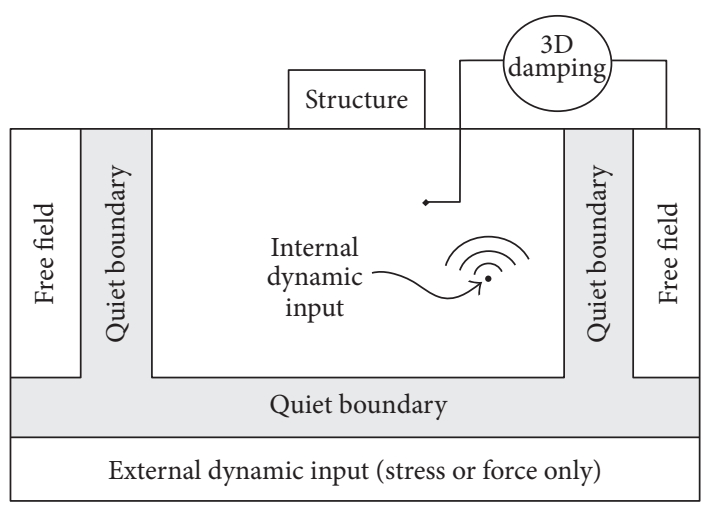

(a)

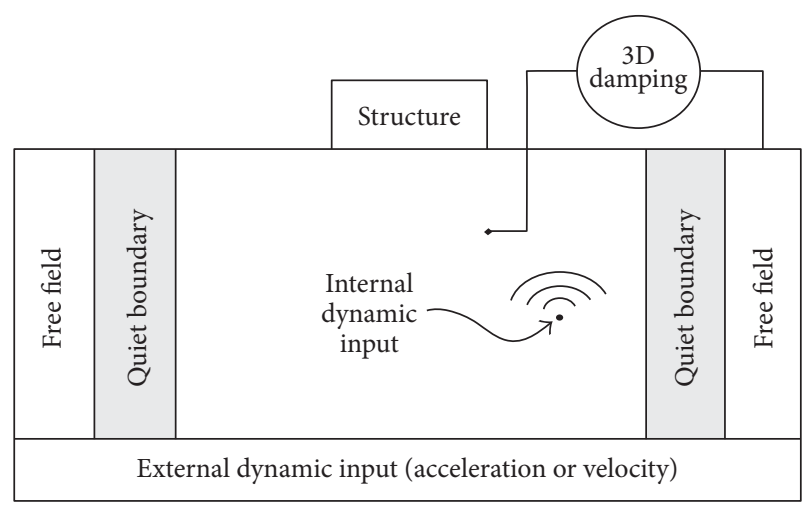

(b)

Figure 7: Quiet boundaries for (a) rigid and (b) flexible beds in FLAC 3D [16].

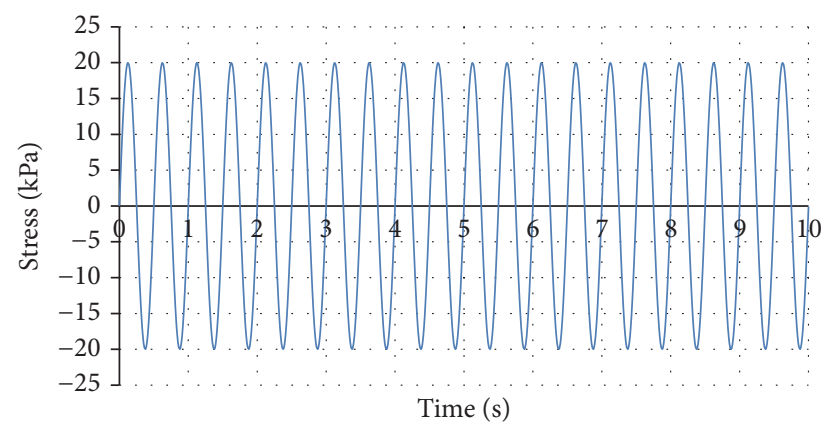

Figure 8: Exerted horizontal stress wave.

\section{Validation of Numerical Model}

In order to further study and investigate the role of each of the concerning parameters, the previously well-validated threedimensional finite difference modeling procedure (previously applied by the corresponding author of the paper) is used [6]. This model was validated using a $1 \mathrm{~g}$ physical model test on medium dense sand with the soil and pile geometry shown in Figure 9. In addition, the adopted mesh along with the comparison of physical modeling results with the results of numerical modeling are also shown in Figure 9. As it can be seen, there is an acceptable agreement between the results of three-dimensional nonlinear finite difference modeling and $1 \mathrm{~g}$ physical modeling [6].

\section{Parametric Study on Finite Difference Model}

As it was mentioned, the effect of some of the geometrical pile parameters and the loads on the static and combined staticdynamic CPRF's settlements has been studied. Considering three pile patterns (different configurations), two different pile diameters, three ratios for $s / d$, four ratios for $l / d$, and two different static load states, a databank with 144 static data series and 144 dynamic ones has been achieved. Figures
10 and 11 show the results of static and combined staticdynamic settlements for the $3 \times 3 \mathrm{CPRF}$ as a sample of the results. As shown, F-architecture is the best architecture, in which the minimum settlements are observed in both static and coupled static-dynamic models. Besides, increasing $s / d$ ratio will result in an increase in the calculated settlements. However, piles with larger lengths cause smaller induced static and coupled static-dynamic settlements. The combined effects of $s / d$ and $l / d$ can be better tracked using a sensitivity analysis. The following sections present the sensitivity analysis, which shows the relative importance of increasing $l / d$ and decreasing $s / d$ in the reduction of induced settlements. As shown and comparing to the effect of increasing $l / d$ ratio, decreasing s/d ratio plays more important role in the reduction of the settlements in both cases.

Furthermore, Tables 1 and 2, respectively, present the results of parametric study on the whole affecting parameters for static loading condition and combined static-dynamic loading condition.

The following subsections present parametric studies conducted on different concerning parameters (e.g., piles' lengths, diameters, spacing, architecture, and axial loads). In this regard and as sample cases, settlements of CPRFs under coupled static-dynamic loads are presented and discussed for different parameters.

4.1. Effect of Piles' Architecture (Pattern) on the Settlements of CPRF. To investigate the effect of piles' arrangement and their architecture on the static and coupled static-dynamic settlements, three different patterns are considered, where $\mathrm{N}$, $\mathrm{F}$, and $\mathrm{M}$ symbols stand for the near-, far-, and mediumdistance piles architectures, respectively. As described, 0.3 and 0.5 meters are the values of piles' diameter used to calculate the induced settlements. In this section, effects of different piles' architectures on the coupled static-dynamic settlements of 0.5 meters in diameter piles under $60 \mathrm{kPa}$ static axial load are evaluated. Figure 12(a) shows coupled staticdynamic settlements of the studied $3 * 3$-CPRF for N, F, and $\mathrm{M}$ arrangements. Also, Figures 12(b) and 12(c), respectively, present corresponding results for $4 * 4$ and $5 * 5$ CPRFs. It 

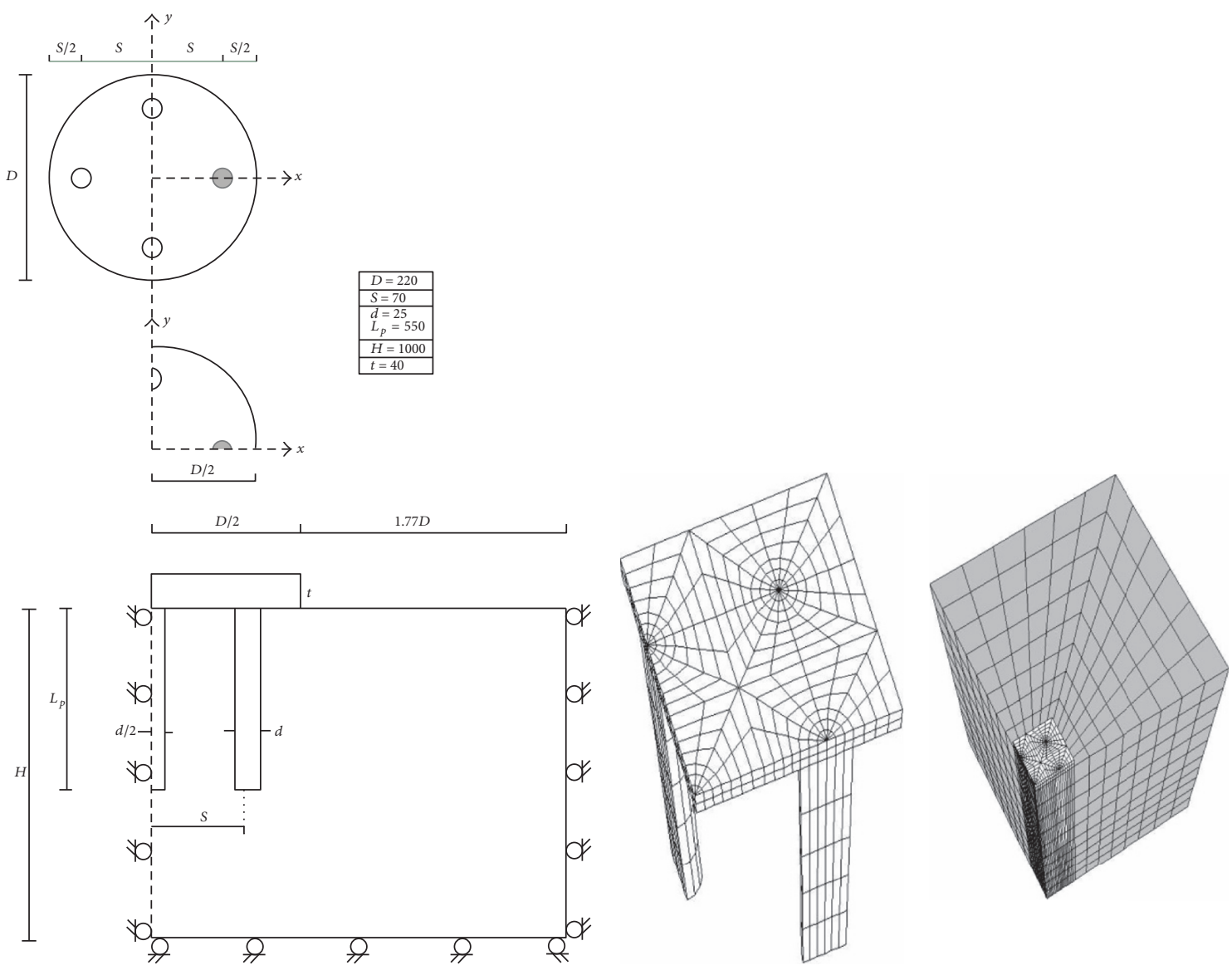

(a)

(b)

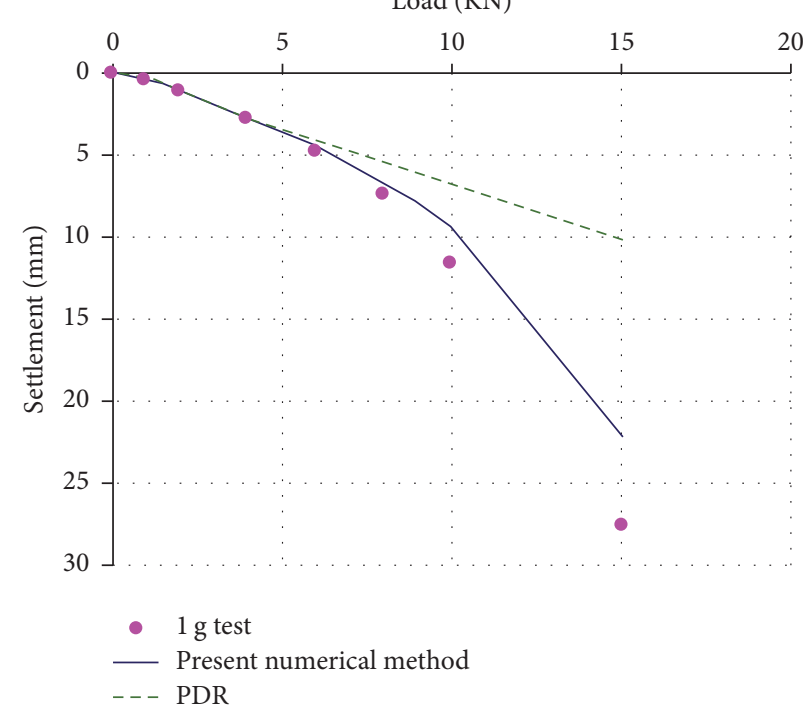

(c)

FIGURE 9: (a) Geometry of conducted physical model. (b) Three-dimensional finite difference mesh. (c) Comparison of the results of finite difference and physical model [6]. 
TABLE 1: Parametric study on the whole affecting parameters for static loading condition.

\begin{tabular}{|c|c|c|c|c|c|}
\hline Number of piles & $d(\mathrm{~m})$ & $s / d$ & $l / d$ & $p(\mathrm{kPa})$ & $S(\mathrm{~cm})$ \\
\hline Mean: 16.67 & Mean: 0.4 & Mean: 5 & Mean: 23 & Mean: 75 & Mean: 5.96 \\
\hline Std. Dev.: 6.57 & Std. Dev.: 0.1 & Std. Dev.: 0.82 & Std. Dev.: 5.94 & Std. Dev.: 15.05 & Std. Dev.: 1.72 \\
\hline 9 & 0.3 & 4 & 16 & 90 & 8.32 \\
\hline 9 & 0.3 & 4 & 20 & 90 & 7.23 \\
\hline 9 & 0.3 & 4 & 24 & 90 & 6.09 \\
\hline 9 & 0.3 & 4 & 32 & 90 & 5 \\
\hline 9 & 0.3 & 5 & 16 & 90 & 7.52 \\
\hline 9 & 0.3 & 5 & 20 & 90 & 6.52 \\
\hline 9 & 0.3 & 5 & 24 & 90 & 5.25 \\
\hline 9 & 0.3 & 5 & 32 & 90 & 4.23 \\
\hline 9 & 0.3 & 6 & 16 & 90 & 7.12 \\
\hline 9 & 0.3 & 6 & 20 & 90 & 6.11 \\
\hline 9 & 0.3 & 6 & 24 & 90 & 5.01 \\
\hline 9 & 0.3 & 6 & 32 & 90 & 4 \\
\hline 9 & 0.3 & 4 & 16 & 60 & 5.83 \\
\hline 9 & 0.3 & 4 & 20 & 60 & 5.03 \\
\hline 9 & 0.3 & 4 & 24 & 60 & 4.31 \\
\hline 9 & 0.3 & 4 & 32 & 60 & 3.5 \\
\hline 9 & 0.3 & 5 & 16 & 60 & 5.32 \\
\hline 9 & 0.3 & 5 & 20 & 60 & 4.66 \\
\hline 9 & 0.3 & 5 & 24 & 60 & 3.71 \\
\hline 9 & 0.3 & 5 & 32 & 60 & 2.961 \\
\hline 9 & 0.3 & 6 & 16 & 60 & 5.02 \\
\hline 9 & 0.3 & 6 & 20 & 60 & 4.31 \\
\hline 9 & 0.3 & 6 & 24 & 60 & 3.51 \\
\hline 9 & 0.3 & 6 & 32 & 60 & 2.8 \\
\hline 16 & 0.3 & 4 & 16 & 90 & 9.152 \\
\hline 16 & 0.3 & 4 & 20 & 90 & 7.953 \\
\hline 16 & 0.3 & 4 & 24 & 90 & 6.699 \\
\hline 16 & 0.3 & 4 & 32 & 90 & 5.5 \\
\hline 16 & 0.3 & 5 & 16 & 90 & 8.272 \\
\hline 16 & 0.3 & 5 & 20 & 90 & 7.172 \\
\hline 16 & 0.3 & 5 & 24 & 90 & 5.775 \\
\hline 16 & 0.3 & 5 & 32 & 90 & 4.653 \\
\hline 16 & 0.3 & 6 & 16 & 90 & 7.832 \\
\hline 16 & 0.3 & 6 & 20 & 90 & 6.72 \\
\hline 16 & 0.3 & 6 & 24 & 90 & 5.51 \\
\hline 16 & 0.3 & 6 & 32 & 90 & 4.4 \\
\hline 16 & 0.3 & 4 & 16 & 60 & 6.864 \\
\hline 16 & 0.3 & 4 & 20 & 60 & 5.96475 \\
\hline 16 & 0.3 & 4 & 24 & 60 & 5.02 \\
\hline 16 & 0.3 & 4 & 32 & 60 & 4.125 \\
\hline 16 & 0.3 & 5 & 16 & 60 & 6.204 \\
\hline 16 & 0.3 & 5 & 20 & 60 & 5.38 \\
\hline 16 & 0.3 & 5 & 24 & 60 & 4.33 \\
\hline 16 & 0.3 & 5 & 32 & 60 & 3.49 \\
\hline 16 & 0.3 & 6 & 16 & 60 & 5.87 \\
\hline 16 & 0.3 & 6 & 20 & 60 & 5.04 \\
\hline 16 & 0.3 & 6 & 24 & 60 & 4.13 \\
\hline 16 & 0.3 & 6 & 32 & 60 & 3.3 \\
\hline 25 & 0.3 & 4 & 16 & 90 & 9.98 \\
\hline 25 & 0.3 & 4 & 20 & 90 & 8.67 \\
\hline 25 & 0.3 & 4 & 24 & 90 & 7.3 \\
\hline
\end{tabular}


TABLe 1: Continued.

\begin{tabular}{|c|c|c|c|c|c|}
\hline Number of piles & $d(\mathrm{~m})$ & $s / d$ & $l / d$ & $p(\mathrm{kPa})$ & $S(\mathrm{~cm})$ \\
\hline Mean: 16.67 & Mean: 0.4 & Mean: 5 & Mean: 23 & Mean: 75 & Mean: 5.96 \\
\hline Std. Dev.: 6.57 & Std. Dev.: 0.1 & Std. Dev.: 0.82 & Std. Dev.: 5.94 & Std. Dev.: 15.05 & Std. Dev.: 1.72 \\
\hline 25 & 0.3 & 4 & 32 & 90 & 5.995 \\
\hline 25 & 0.3 & 5 & 16 & 90 & 9.02 \\
\hline 25 & 0.3 & 5 & 20 & 90 & 7.82 \\
\hline 25 & 0.3 & 5 & 24 & 90 & 6.29 \\
\hline 25 & 0.3 & 5 & 32 & 90 & 5.07 \\
\hline 25 & 0.3 & 6 & 16 & 90 & 8.54 \\
\hline 25 & 0.3 & 6 & 20 & 90 & 7.33 \\
\hline 25 & 0.3 & 6 & 24 & 90 & 6.01 \\
\hline 25 & 0.3 & 6 & 32 & 90 & 4.8 \\
\hline 25 & 0.3 & 4 & 16 & 60 & 7.78 \\
\hline 25 & 0.3 & 4 & 20 & 60 & 6.76 \\
\hline 25 & 0.3 & 4 & 24 & 60 & 5.7 \\
\hline 25 & 0.3 & 4 & 32 & 60 & 4.68 \\
\hline 25 & 0.3 & 5 & 16 & 60 & 7.03 \\
\hline 25 & 0.3 & 5 & 20 & 60 & 6.1 \\
\hline 25 & 0.3 & 5 & 24 & 60 & 4.91 \\
\hline 25 & 0.3 & 5 & 32 & 60 & 3.96 \\
\hline 25 & 0.3 & 6 & 16 & 60 & 6.66 \\
\hline 25 & 0.3 & 6 & 20 & 60 & 5.71 \\
\hline 25 & 0.3 & 6 & 24 & 60 & 4.69 \\
\hline 25 & 0.3 & 6 & 32 & 60 & 3.74 \\
\hline 9 & 0.5 & 4 & 16 & 90 & 9.01 \\
\hline 9 & 0.5 & 4 & 20 & 90 & 8.29 \\
\hline 9 & 0.5 & 4 & 24 & 90 & 6.72 \\
\hline 9 & 0.5 & 4 & 32 & 90 & 5.23 \\
\hline 9 & 0.5 & 5 & 16 & 90 & 8.11 \\
\hline 9 & 0.5 & 5 & 20 & 90 & 7 \\
\hline 9 & 0.5 & 5 & 24 & 90 & 5.72 \\
\hline 9 & 0.5 & 5 & 32 & 90 & 4.75 \\
\hline 9 & 0.5 & 6 & 16 & 90 & 7.52 \\
\hline 9 & 0.5 & 6 & 20 & 90 & 6.82 \\
\hline 9 & 0.5 & 6 & 24 & 90 & 5.56 \\
\hline 9 & 0.5 & 6 & 32 & 90 & 4.52 \\
\hline 9 & 0.5 & 4 & 16 & 60 & 5.95 \\
\hline 9 & 0.5 & 4 & 20 & 60 & 5.47 \\
\hline 9 & 0.5 & 4 & 24 & 60 & 4.44 \\
\hline 9 & 0.5 & 4 & 32 & 60 & 3.45 \\
\hline 9 & 0.5 & 5 & 16 & 60 & 5.35 \\
\hline 9 & 0.5 & 5 & 20 & 60 & 4.62 \\
\hline 9 & 0.5 & 5 & 24 & 60 & 3.78 \\
\hline 9 & 0.5 & 5 & 32 & 60 & 3.14 \\
\hline 9 & 0.5 & 6 & 16 & 60 & 4.96 \\
\hline 9 & 0.5 & 6 & 20 & 60 & 4.5 \\
\hline 9 & 0.5 & 6 & 24 & 60 & 3.67 \\
\hline 9 & 0.5 & 6 & 32 & 60 & 2.98 \\
\hline 16 & 0.5 & 4 & 16 & 90 & 9.73 \\
\hline 16 & 0.5 & 4 & 20 & 90 & 8.95 \\
\hline 16 & 0.5 & 4 & 24 & 90 & 7.26 \\
\hline 16 & 0.5 & 4 & 32 & 90 & 5.65 \\
\hline 16 & 0.5 & 5 & 16 & 90 & 8.76 \\
\hline 16 & 0.5 & 5 & 20 & 90 & 7.56 \\
\hline
\end{tabular}


TABle 1: Continued.

\begin{tabular}{|c|c|c|c|c|c|}
\hline Number of piles & $d(\mathrm{~m})$ & $s / d$ & $l / d$ & $p(\mathrm{kPa})$ & $S(\mathrm{~cm})$ \\
\hline Mean: 16.67 & Mean: 0.4 & Mean: 5 & Mean: 23 & Mean: 75 & Mean: 5.96 \\
\hline Std. Dev.: 6.57 & Std. Dev.: 0.1 & Std. Dev.: 0.82 & Std. Dev.: 5.94 & Std. Dev.: 15.05 & Std. Dev.: 1.72 \\
\hline 16 & 0.5 & 5 & 24 & 90 & 6.18 \\
\hline 16 & 0.5 & 5 & 32 & 90 & 5.13 \\
\hline 16 & 0.5 & 6 & 16 & 90 & 8.12 \\
\hline 16 & 0.5 & 6 & 20 & 90 & 7.37 \\
\hline 16 & 0.5 & 6 & 24 & 90 & 6 \\
\hline 16 & 0.5 & 6 & 32 & 90 & 4.88 \\
\hline 16 & 0.5 & 4 & 16 & 60 & 6.81 \\
\hline 16 & 0.5 & 4 & 20 & 60 & 6.27 \\
\hline 16 & 0.5 & 4 & 24 & 60 & 5.08 \\
\hline 16 & 0.5 & 4 & 32 & 60 & 3.95 \\
\hline 16 & 0.5 & 5 & 16 & 60 & 6.13 \\
\hline 16 & 0.5 & 5 & 20 & 60 & 5.29 \\
\hline 16 & 0.5 & 5 & 24 & 60 & 4.32 \\
\hline 16 & 0.5 & 5 & 32 & 60 & 3.59 \\
\hline 16 & 0.5 & 6 & 16 & 60 & 5.69 \\
\hline 16 & 0.5 & 6 & 20 & 60 & 5.16 \\
\hline 16 & 0.5 & 6 & 24 & 60 & 4.2 \\
\hline 16 & 0.5 & 6 & 32 & 60 & 3.42 \\
\hline 25 & 0.5 & 4 & 16 & 90 & 10.7 \\
\hline 25 & 0.5 & 4 & 20 & 90 & 9.85 \\
\hline 25 & 0.5 & 4 & 24 & 90 & 7.98 \\
\hline 25 & 0.5 & 4 & 32 & 90 & 6.21 \\
\hline 25 & 0.5 & 5 & 16 & 90 & 9.63 \\
\hline 25 & 0.5 & 5 & 20 & 90 & 8.32 \\
\hline 25 & 0.5 & 5 & 24 & 90 & 6.8 \\
\hline 25 & 0.5 & 5 & 32 & 90 & 5.64 \\
\hline 25 & 0.5 & 6 & 16 & 90 & 8.93 \\
\hline 25 & 0.5 & 6 & 20 & 90 & 8.1 \\
\hline 25 & 0.5 & 6 & 24 & 90 & 6.61 \\
\hline 25 & 0.5 & 6 & 32 & 90 & 5.37 \\
\hline 25 & 0.5 & 4 & 16 & 60 & 7.92 \\
\hline 25 & 0.5 & 4 & 20 & 60 & 7.29 \\
\hline 25 & 0.5 & 4 & 24 & 60 & 5.91 \\
\hline 25 & 0.5 & 4 & 32 & 60 & 4.6 \\
\hline 25 & 0.5 & 5 & 16 & 60 & 7.13 \\
\hline 25 & 0.5 & 5 & 20 & 60 & 6.15 \\
\hline 25 & 0.5 & 5 & 24 & 60 & 5.03 \\
\hline 25 & 0.5 & 5 & 32 & 60 & 4.18 \\
\hline 25 & 0.5 & 6 & 16 & 60 & 6.61 \\
\hline 25 & 0.5 & 6 & 20 & 60 & 6 \\
\hline 25 & 0.5 & 6 & 24 & 60 & 4.89 \\
\hline 25 & 0.5 & 6 & 32 & 60 & 3.97 \\
\hline
\end{tabular}

should be noted that the reported value for the settlement of each CPRF is the average of pile tip's settlement since a rigid behavior is assumed for the piles' cap.

As shown, $\mathrm{N}$-architecture CPRFs experience the largest settlements, while F-architecture shows a better resistance against settlements. This is because of the piles group's performance in the CPRF. Indeed, in far-piles architecture (F), with decreasing the relative effect of piles, each individual pile behaves as a single pile and enhances the resistance of the whole CPRF. Nevertheless, in the $\mathrm{N}$-architecture, overlapping stress bubbles of individual piles, the maximum resistance of the whole CPRF against the settlement decreases. 
TABLE 2: Parametric study on the whole affecting parameters for combined static-dynamic loading condition.

\begin{tabular}{|c|c|c|c|c|c|}
\hline Number of piles & $d(\mathrm{~m})$ & $s / d$ & $l / d$ & $p(\mathrm{kPa})$ & $S(\mathrm{~cm})$ \\
\hline Mean: 16.67 & Mean: 0.4 & Mean: 5 & Mean: 23 & Mean: 75 & Mean: 6.67 \\
\hline Std. Dev.: 6.57 & Std. Dev.: 0.1 & Std. Dev.: 0.82 & Std. Dev.: 5.94 & Std. Dev.: 15.05 & Std. Dev.: 2.21 \\
\hline 9 & 0.3 & 4 & 16 & 90 & 10.816 \\
\hline 9 & 0.3 & 4 & 20 & 90 & 9.04 \\
\hline 9 & 0.3 & 4 & 24 & 90 & 6.94 \\
\hline 9 & 0.3 & 4 & 32 & 90 & 5.5 \\
\hline 9 & 0.3 & 5 & 16 & 90 & 9.1744 \\
\hline 9 & 0.3 & 5 & 20 & 90 & 7.76 \\
\hline 9 & 0.3 & 5 & 24 & 90 & 5.78 \\
\hline 9 & 0.3 & 5 & 32 & 90 & 4.48 \\
\hline 9 & 0.3 & 6 & 16 & 90 & 8.4728 \\
\hline 9 & 0.3 & 6 & 20 & 90 & 7.03 \\
\hline 9 & 0.3 & 6 & 24 & 90 & 5.41 \\
\hline 9 & 0.3 & 6 & 32 & 90 & 4.16 \\
\hline 9 & 0.3 & 4 & 16 & 60 & 7.35 \\
\hline 9 & 0.3 & 4 & 20 & 60 & 6.04 \\
\hline 9 & 0.3 & 4 & 24 & 60 & 4.78 \\
\hline 9 & 0.3 & 4 & 32 & 60 & 3.68 \\
\hline 9 & 0.3 & 5 & 16 & 60 & 6.33 \\
\hline 9 & 0.3 & 5 & 20 & 60 & 5.36 \\
\hline 9 & 0.3 & 5 & 24 & 60 & 3.97 \\
\hline 9 & 0.3 & 5 & 32 & 60 & 3.02 \\
\hline 9 & 0.3 & 6 & 16 & 60 & 5.82 \\
\hline 9 & 0.3 & 6 & 20 & 60 & 4.83 \\
\hline 9 & 0.3 & 6 & 24 & 60 & 3.65 \\
\hline 9 & 0.3 & 6 & 32 & 60 & 2.83 \\
\hline 16 & 0.3 & 4 & 16 & 90 & 11.62 \\
\hline 16 & 0.3 & 4 & 20 & 90 & 9.54 \\
\hline 16 & 0.3 & 4 & 24 & 90 & 7.37 \\
\hline 16 & 0.3 & 4 & 32 & 90 & 5.78 \\
\hline 16 & 0.3 & 5 & 16 & 90 & 9.93 \\
\hline 16 & 0.3 & 5 & 20 & 90 & 8.32 \\
\hline 16 & 0.3 & 5 & 24 & 90 & 6.24 \\
\hline 16 & 0.3 & 5 & 32 & 90 & 4.79 \\
\hline 16 & 0.3 & 6 & 16 & 90 & 9.16 \\
\hline 16 & 0.3 & 6 & 20 & 90 & 7.66 \\
\hline 16 & 0.3 & 6 & 24 & 90 & 5.62 \\
\hline 16 & 0.3 & 6 & 32 & 90 & 4.44 \\
\hline 16 & 0.3 & 4 & 16 & 60 & 8.65 \\
\hline 16 & 0.3 & 4 & 20 & 60 & 7.16 \\
\hline 16 & 0.3 & 4 & 24 & 60 & 5.58 \\
\hline 16 & 0.3 & 4 & 32 & 60 & 4.33 \\
\hline 16 & 0.3 & 5 & 16 & 60 & 7.38 \\
\hline 16 & 0.3 & 5 & 20 & 60 & 6.19 \\
\hline 16 & 0.3 & 5 & 24 & 60 & 4.63 \\
\hline 16 & 0.3 & 5 & 32 & 60 & 3.56 \\
\hline 16 & 0.3 & 6 & 16 & 60 & 6.81 \\
\hline 16 & 0.3 & 6 & 20 & 60 & 5.65 \\
\hline 16 & 0.3 & 6 & 24 & 60 & 4.3 \\
\hline 16 & 0.3 & 6 & 32 & 60 & 3.33 \\
\hline 25 & 0.3 & 4 & 16 & 90 & 11.77 \\
\hline 25 & 0.3 & 4 & 20 & 90 & 9.88 \\
\hline
\end{tabular}


TABLE 2: Continued.

\begin{tabular}{|c|c|c|c|c|c|}
\hline Number of piles & $d(\mathrm{~m})$ & $s / d$ & $l / d$ & $p(\mathrm{kPa})$ & $S(\mathrm{~cm})$ \\
\hline Mean: 16.67 & Mean: 0.4 & Mean: 5 & Mean: 23 & Mean: 75 & Mean: 6.67 \\
\hline Std. Dev.: 6.57 & Std. Dev.: 0.1 & Std. Dev.: 0.82 & Std. Dev.: 5.94 & Std. Dev.: 15.05 & Std. Dev.: 2.21 \\
\hline 25 & 0.3 & 4 & 24 & 90 & 8.03 \\
\hline 25 & 0.3 & 4 & 32 & 90 & 6.29 \\
\hline 25 & 0.3 & 5 & 16 & 90 & 10.37 \\
\hline 25 & 0.3 & 5 & 20 & 90 & 8.68 \\
\hline 25 & 0.3 & 5 & 24 & 90 & 6.8 \\
\hline 25 & 0.3 & 5 & 32 & 90 & 5.22 \\
\hline 25 & 0.3 & 6 & 16 & 90 & 9.65 \\
\hline 25 & 0.3 & 6 & 20 & 90 & 8.06 \\
\hline 25 & 0.3 & 6 & 24 & 90 & 6.13 \\
\hline 25 & 0.3 & 6 & 32 & 90 & 4.84 \\
\hline 25 & 0.3 & 4 & 16 & 60 & 9.8 \\
\hline 25 & 0.3 & 4 & 20 & 60 & 7.91 \\
\hline 25 & 0.3 & 4 & 24 & 60 & 6.38 \\
\hline 25 & 0.3 & 4 & 32 & 60 & 4.91 \\
\hline 25 & 0.3 & 5 & 16 & 60 & 8.37 \\
\hline 25 & 0.3 & 5 & 20 & 60 & 6.83 \\
\hline 25 & 0.3 & 5 & 24 & 60 & 5.35 \\
\hline 25 & 0.3 & 5 & 32 & 60 & 4.04 \\
\hline 25 & 0.3 & 6 & 16 & 60 & 7.66 \\
\hline 25 & 0.3 & 6 & 20 & 60 & 6.29 \\
\hline 25 & 0.3 & 6 & 24 & 60 & 4.87 \\
\hline 25 & 0.3 & 6 & 32 & 60 & 3.78 \\
\hline 9 & 0.5 & 4 & 16 & 90 & 11.3526 \\
\hline 9 & 0.5 & 4 & 20 & 90 & 9.7 \\
\hline 9 & 0.5 & 4 & 24 & 90 & 7.46 \\
\hline 9 & 0.5 & 4 & 32 & 90 & 5.65 \\
\hline 9 & 0.5 & 5 & 16 & 90 & 9.6509 \\
\hline 9 & 0.5 & 5 & 20 & 90 & 7.98 \\
\hline 9 & 0.5 & 5 & 24 & 90 & 6.29 \\
\hline 9 & 0.5 & 5 & 32 & 90 & 4.85 \\
\hline 9 & 0.5 & 6 & 16 & 90 & 8.7232 \\
\hline 9 & 0.5 & 6 & 20 & 90 & 7.5 \\
\hline 9 & 0.5 & 6 & 24 & 90 & 5.84 \\
\hline 9 & 0.5 & 6 & 32 & 90 & 4.57 \\
\hline 9 & 0.5 & 4 & 16 & 60 & 7.2 \\
\hline 9 & 0.5 & 4 & 20 & 60 & 6.4 \\
\hline 9 & 0.5 & 4 & 24 & 60 & 4.83 \\
\hline 9 & 0.5 & 4 & 32 & 60 & 3.62 \\
\hline 9 & 0.5 & 5 & 16 & 60 & 6.16 \\
\hline 9 & 0.5 & 5 & 20 & 60 & 5.17 \\
\hline 9 & 0.5 & 5 & 24 & 60 & 3.96 \\
\hline 9 & 0.5 & 5 & 32 & 60 & 3.2 \\
\hline 9 & 0.5 & 6 & 16 & 60 & 5.71 \\
\hline 9 & 0.5 & 6 & 20 & 60 & 4.95 \\
\hline 9 & 0.5 & 6 & 24 & 60 & 3.74 \\
\hline 9 & 0.5 & 6 & 32 & 60 & 3.01 \\
\hline 16 & 0.5 & 4 & 16 & 90 & 11.87 \\
\hline 16 & 0.5 & 4 & 20 & 90 & 10.56 \\
\hline 16 & 0.5 & 4 & 24 & 90 & 7.77 \\
\hline 16 & 0.5 & 4 & 32 & 90 & 5.93 \\
\hline
\end{tabular}


TABle 2: Continued.

\begin{tabular}{|c|c|c|c|c|c|}
\hline Number of piles & $d(\mathrm{~m})$ & $s / d$ & $l / d$ & $p(\mathrm{kPa})$ & $S(\mathrm{~cm})$ \\
\hline Mean: 16.67 & Mean: 0.4 & Mean: 5 & Mean: 23 & Mean: 75 & Mean: 6.67 \\
\hline Std. Dev.: 6.57 & Std. Dev.: 0.1 & Std. Dev.: 0.82 & Std. Dev.: 5.94 & Std. Dev.: 15.05 & Std. Dev.: 2.21 \\
\hline 16 & 0.5 & 5 & 16 & 90 & 10.25 \\
\hline 16 & 0.5 & 5 & 20 & 90 & 8.69 \\
\hline 16 & 0.5 & 5 & 24 & 90 & 6.42 \\
\hline 16 & 0.5 & 5 & 32 & 90 & 5.28 \\
\hline 16 & 0.5 & 6 & 16 & 90 & 9.42 \\
\hline 16 & 0.5 & 6 & 20 & 90 & 8.25 \\
\hline 16 & 0.5 & 6 & 24 & 90 & 6.12 \\
\hline 16 & 0.5 & 6 & 32 & 90 & 4.93 \\
\hline 16 & 0.5 & 4 & 16 & 60 & 8.24 \\
\hline 16 & 0.5 & 4 & 20 & 60 & 7.4 \\
\hline 16 & 0.5 & 4 & 24 & 60 & 5.59 \\
\hline 16 & 0.5 & 4 & 32 & 60 & 4.15 \\
\hline 16 & 0.5 & 5 & 16 & 60 & 7.11 \\
\hline 16 & 0.5 & 5 & 20 & 60 & 5.93 \\
\hline 16 & 0.5 & 5 & 24 & 60 & 4.54 \\
\hline 16 & 0.5 & 5 & 32 & 60 & 3.66 \\
\hline 16 & 0.5 & 6 & 16 & 60 & 6.54 \\
\hline 16 & 0.5 & 6 & 20 & 60 & 5.67 \\
\hline 16 & 0.5 & 6 & 24 & 60 & 4.37 \\
\hline 16 & 0.5 & 6 & 32 & 60 & 3.45 \\
\hline 25 & 0.5 & 4 & 16 & 90 & 12.31 \\
\hline 25 & 0.5 & 4 & 20 & 90 & 10.83 \\
\hline 25 & 0.5 & 4 & 24 & 90 & 8.46 \\
\hline 25 & 0.5 & 4 & 32 & 90 & 6.34 \\
\hline 25 & 0.5 & 5 & 16 & 90 & 10.79 \\
\hline 25 & 0.5 & 5 & 20 & 90 & 9.23 \\
\hline 25 & 0.5 & 5 & 24 & 90 & 7.14 \\
\hline 25 & 0.5 & 5 & 32 & 90 & 5.81 \\
\hline 25 & 0.5 & 6 & 16 & 90 & 10.01 \\
\hline 25 & 0.5 & 6 & 20 & 90 & 8.59 \\
\hline 25 & 0.5 & 6 & 24 & 90 & 6.74 \\
\hline 25 & 0.5 & 6 & 32 & 90 & 5.42 \\
\hline 25 & 0.5 & 4 & 16 & 60 & 9.58 \\
\hline 25 & 0.5 & 4 & 20 & 60 & 8.38 \\
\hline 25 & 0.5 & 4 & 24 & 60 & 6.5 \\
\hline 25 & 0.5 & 4 & 32 & 60 & 4.69 \\
\hline 25 & 0.5 & 5 & 16 & 60 & 8.27 \\
\hline 25 & 0.5 & 5 & 20 & 60 & 6.77 \\
\hline 25 & 0.5 & 5 & 24 & 60 & 5.33 \\
\hline 25 & 0.5 & 5 & 32 & 60 & 4.26 \\
\hline 25 & 0.5 & 6 & 16 & 60 & 7.47 \\
\hline 25 & 0.5 & 6 & 20 & 60 & 6.48 \\
\hline 25 & 0.5 & 6 & 24 & 60 & 4.99 \\
\hline 25 & 0.5 & 6 & 32 & 60 & 4.01 \\
\hline
\end{tabular}




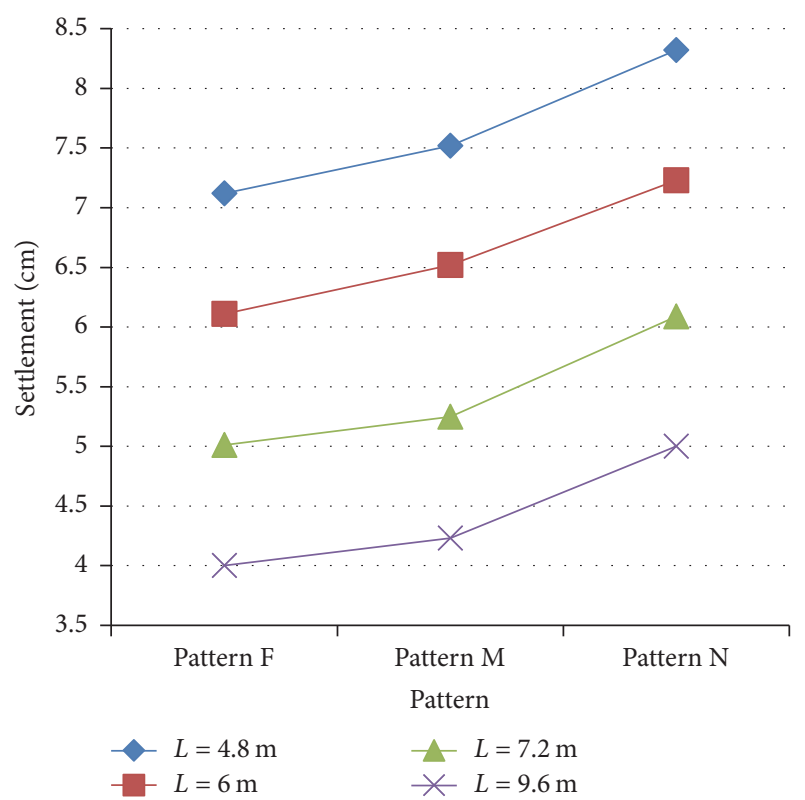

Figure 10: Static CPRF's settlements at $90 \mathrm{kPa}$ overburden.

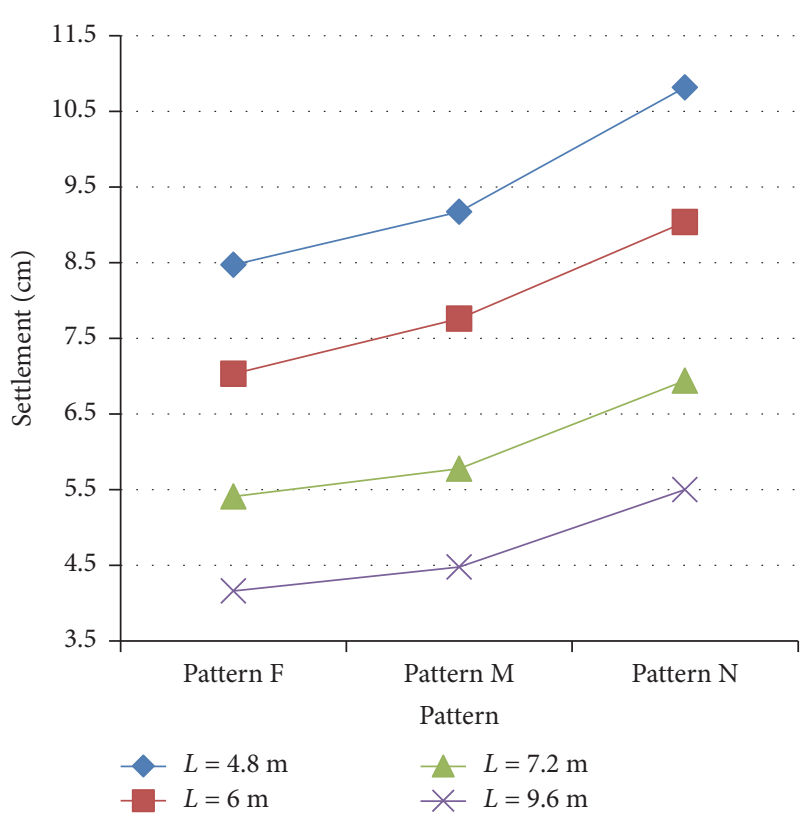

FIGURE 11: CPRF's settlements under combined static $(90 \mathrm{kPa})$ dynamic load.

4.2. Effect of Pile's Length on the Settlements of CPRF. To show the effect of piles' length, as sample results, settlements of different CPRFs are shown in Figure 13. Figures 13(a), 13(b), and $13(\mathrm{c})$, respectively, describe the effect of piles' length on the coupled static-dynamic settlements of the studied $3 * 3$, $4 * 4$, and $5 * 5$ CPRFs under $60 \mathrm{kPa}$ static axial load for 0.5 meters in diameter piles.
It is seen that, for a constant architecture, with increasing the length of piles, value of coupled static-dynamic settlements decreases.

4.3. Effect of Axial Pile Loads on the Settlements of CPRF. In this section, as a sample case, settlements of a $3 * 3$ architecture CPRF (with constant diameter piles, $d=0.5 \mathrm{~m}$ ) under $60 \mathrm{kPa}$ and $90 \mathrm{kPa}$ static axial force subjected to a horizontal dynamic load are evaluated. As shown in Figure 14, the value of axial force affects the induced settlements. It is observed that, with increasing the static axial force, the induced settlements increase.

\section{Artificial Intelligence Techniques}

5.1. Artificial Neural Networks. Artificial Intelligence has different branches. Among these branches, Artificial Neural Networks (ANNs) are known as the black box, while Evolutionary Polynomial regression (EPR) is referred to as the grey box method. In ANNs, generally, experimental datasets are used to attain relationships between input and output parameters. In this method, the large number of databases is one of the requirements to get the results with less error [17-22, 37].

The relationship between input and output parameters is gained using the learning rules. In spite of classical statistical methods, neural networks do not need any previous knowledge about the quality and mechanics of the problem and their concerning parameters. One of the most in-demand kinds of neural networks is multilayer perceptron (MLP) networks, which is formed using correct definition of its constructing layers, input, and hidden and output layers [23]. Regarding the type of problem complexity and its nonlinearity, the number of MLP layers is defined [24]. The constructing elements of each layer are neurons, which are connected to the neurons of other layers, but cannot make connection with the neurons of the same layer. The relationship between neurons of a layer to the neurons of the next neighboring layer is carried out using some connections. The analyzed information will be multiplied to the assigned weight of each node and then will be transformed using the activation function [38]. In an MLP, as shown in Figure 15, independent input variables are connected to the neurons of hidden layers and can predict the multiple dependent output variables based on network training (by detecting the similarities between input and output parameters and minimizing the prediction errors) [25]. Hence, architecture of the network, learning rule, and the transfer function are key parameters, which should be correctly defined to build a proper neural network [35].

5.1.1. Neural Network Training. Feed-Forward Backpropagation, FFBP, is the most applied algorithm used to train the neural networks for a broad range of engineering applications [26]. In this training algorithm, firstly and in forward pass, assuming a primary value for connection between neurons, outputs are forecasted and then the computational error is calculated. This error will be backcalculated to update the 


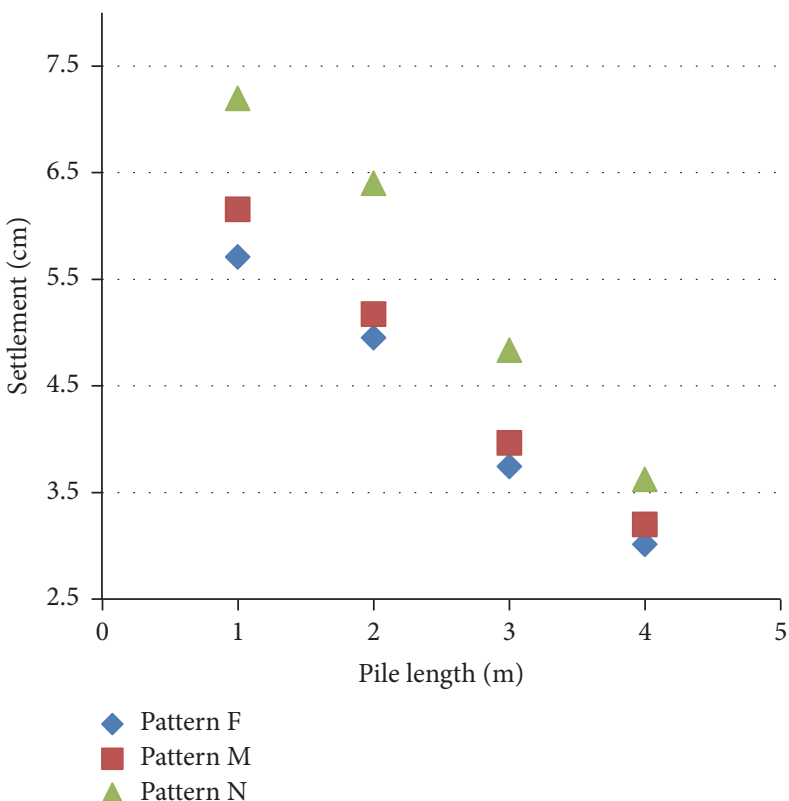

(a) $3 * 3$ piles

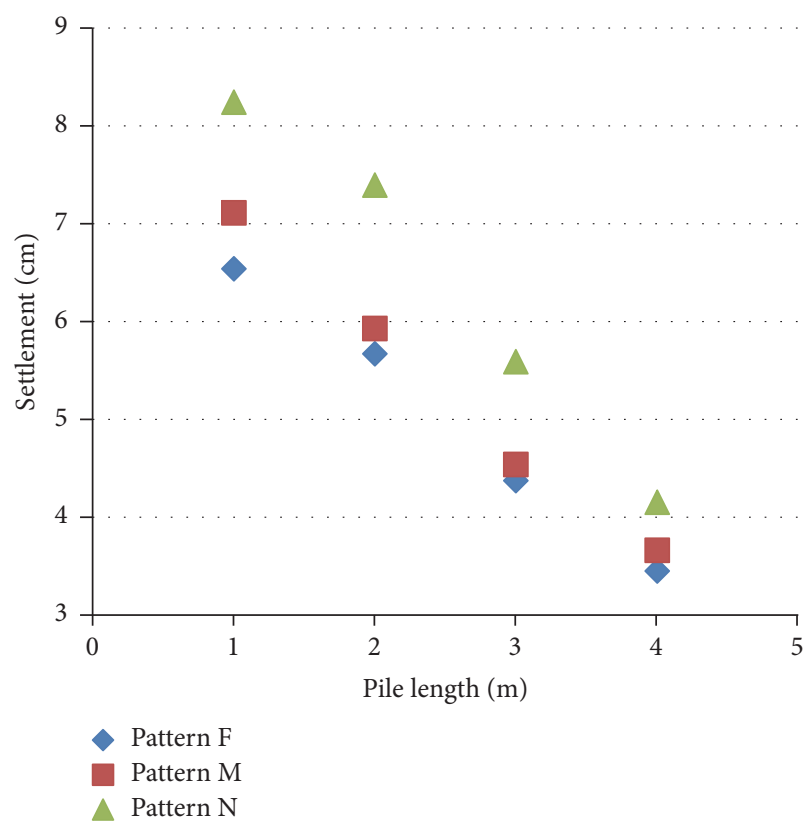

(b) $4 * 4$ piles

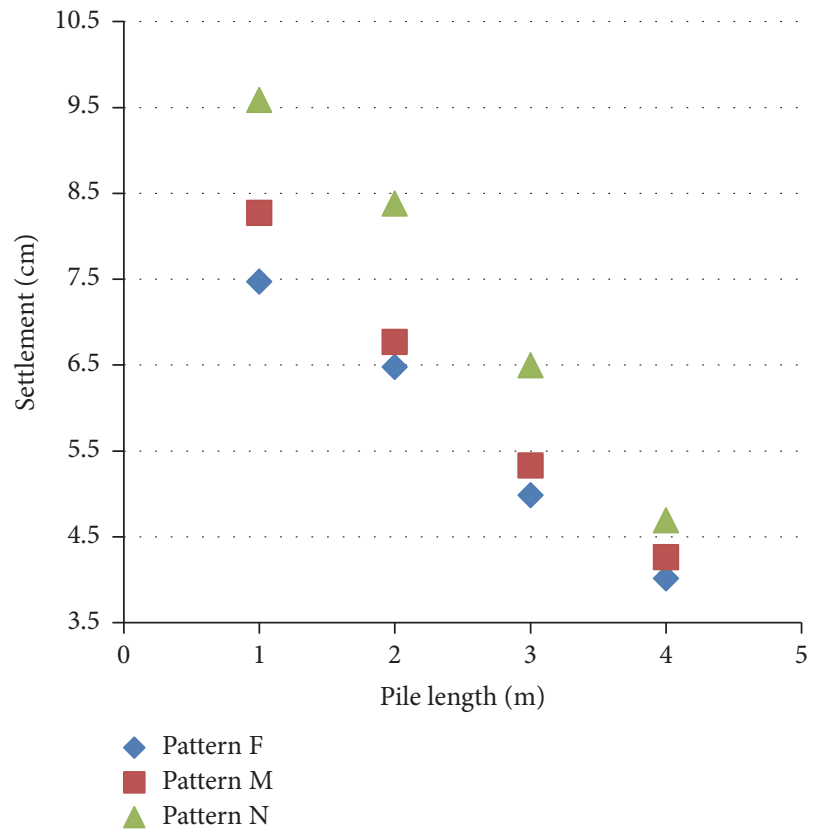

(c) $5 * 5$ piles

FIGURE 12: Effect of piles' architecture on the coupled static-dynamic settlements of CPRF ( $d=0.5 \mathrm{~m}, p=60 \mathrm{kPa})$.

neuron's weights and obtain accurate outputs. This second phase is called backpropagation phase [27].

In order to make all the input and output parameters dimensionless, (7) $[28,29]$ is used and all the parameters are normalized to a $0-1$ scale.

\section{Scaled Parameter}

$$
=\frac{(\text { unscaled parameter }- \text { parameter's min. value })}{(\text { parameter's max. value }- \text { parameter's min. value })} \text {. }
$$

In this paper, training and cross-validation datasets were made through random selection of $85 \%$ of the whole data. The remaining $15 \%$ are the testing data series. Different transfer functions, for example, TANSIG, LOGSIG, and PURELIN, are available to be used as the transfer function to mathematically represent, in terms of spatial or temporal frequency, the relation between the input and output. The transfer functions usually have a sigmoid shape, but they may also take the form of other nonlinear functions, piecewise linear functions, or step functions. Hence, to evaluate the ability of different 


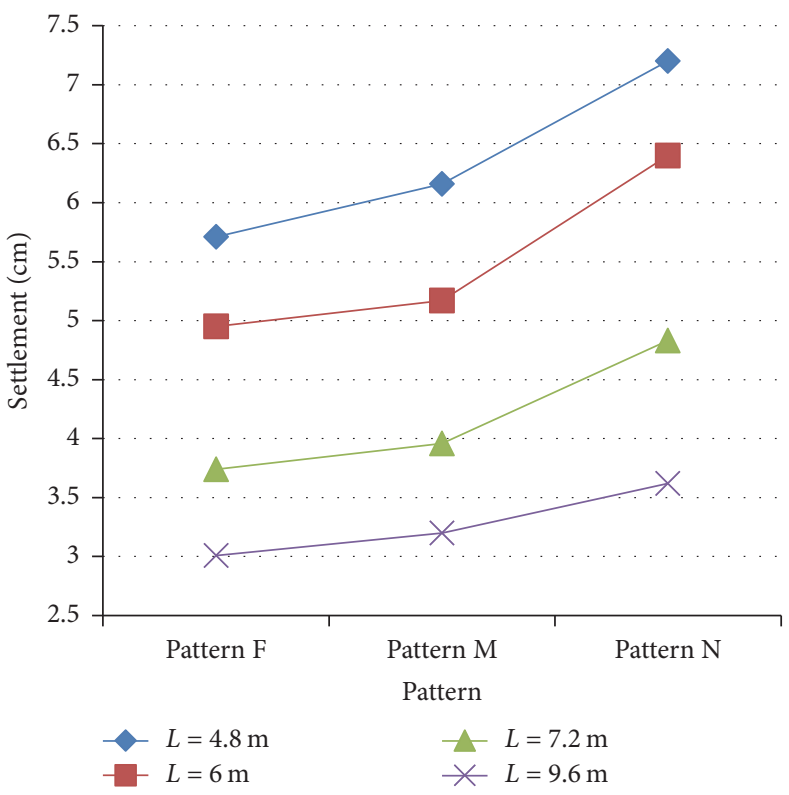

(a) $3 * 3$ piles

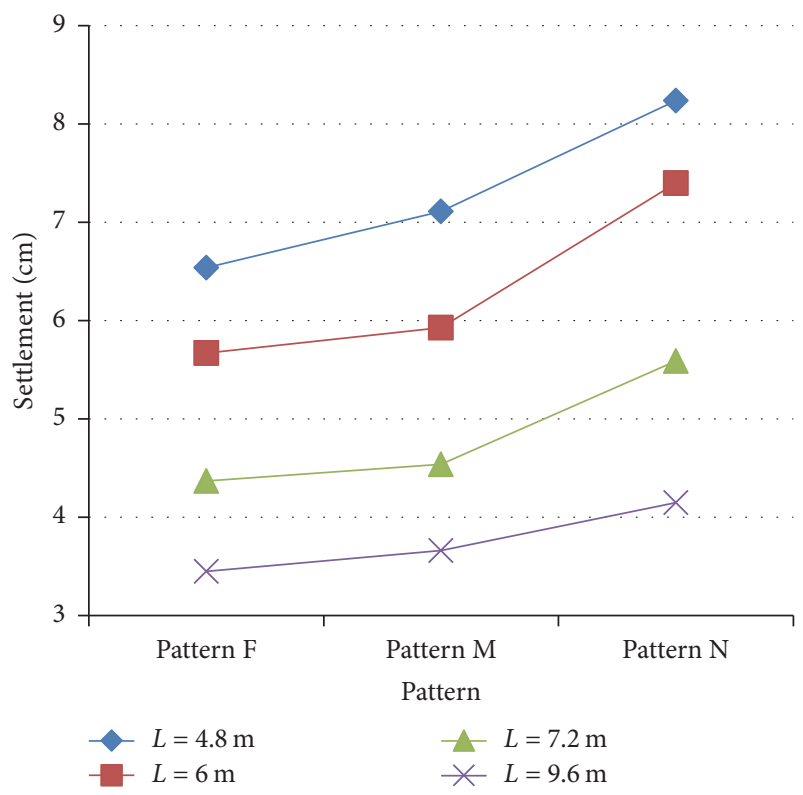

(b) $4 * 4$ piles

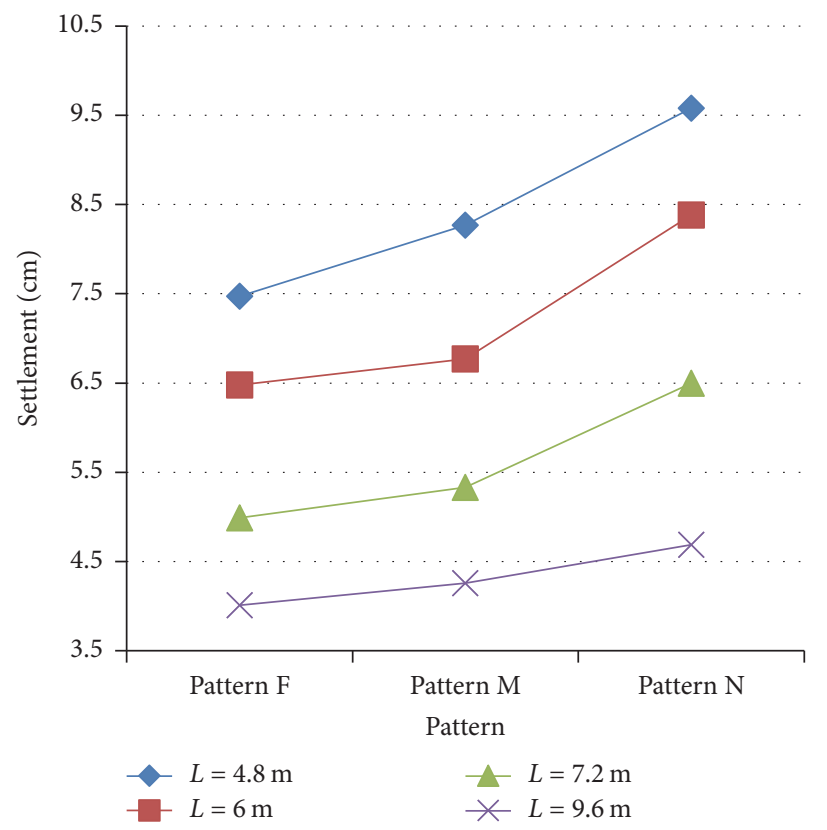

(c) $5 * 5$ piles

FIGURE 13: Effect of piles' length on the coupled static-dynamic settlements of CPRF $(d=0.5 \mathrm{~m}, p=60 \mathrm{kPa})$.

transfer functions in the prediction of coupled static-dynamic CPRF's settlements, the mentioned transfer functions were applied and TANSIG function showing the best performance was selected for the modeling purposes. Figure 16 shows the TANSIG transfer function. Also, (8) shows the formula used to calculate TANSIG function [39].

$$
f=\frac{e^{e_{x}}-e^{-e_{x}}}{e^{e_{x}}+e^{-e_{x}}}
$$

where $e_{x}$ represents the weighted sum for each of the concerning input parameters [39].
In addition, underfitting and overfitting should be avoided during constructing the networks. Indeed, using incorrect number of solution epochs leads to these two phenomena. Overfitting corresponds to the case of too many training cycles, while underfitting declares that insufficient epochs are used to train the network [30].

5.2. Evolutionary Polynomial Regression Modeling. Evolutionary Polynomial Regression (EPR) modeling simultaneously uses the advantages of both numerical regression analysis and the genetic programing. It applies the least 


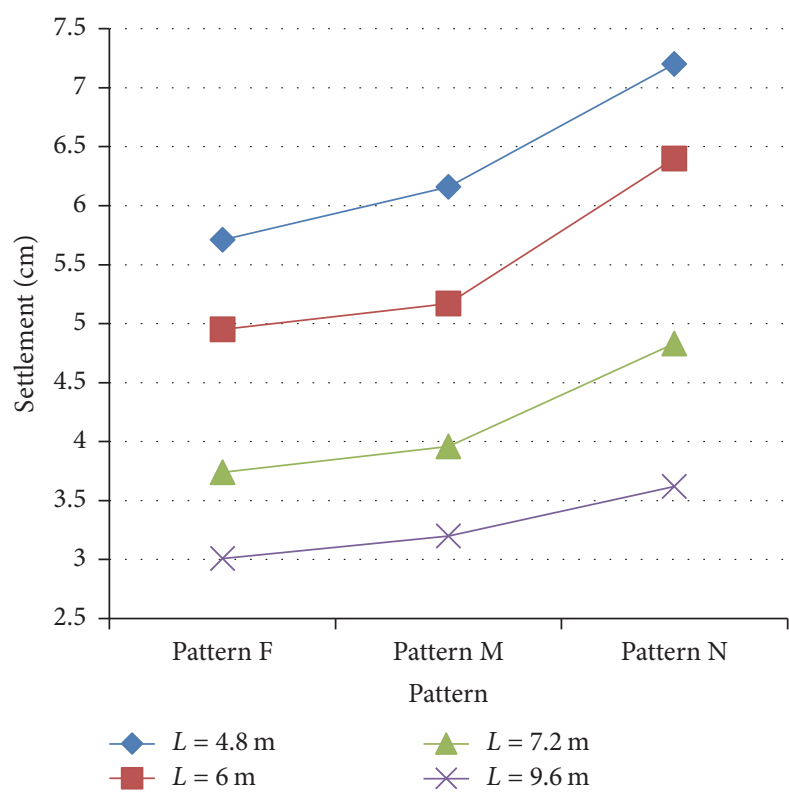

(a) $p=60 \mathrm{kPa}$

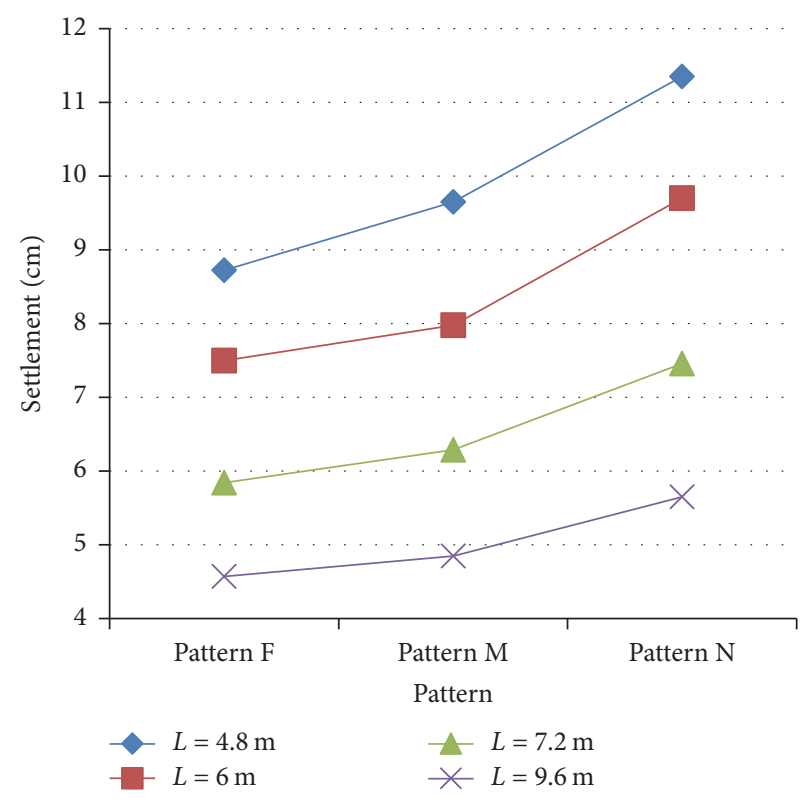

(b) $p=90 \mathrm{kPa}$

FIGURE 14: Effect of axial load on the coupled static-dynamic settlements of CPRF ( $d=0.5 \mathrm{~m}, 3 * 3$ architecture).

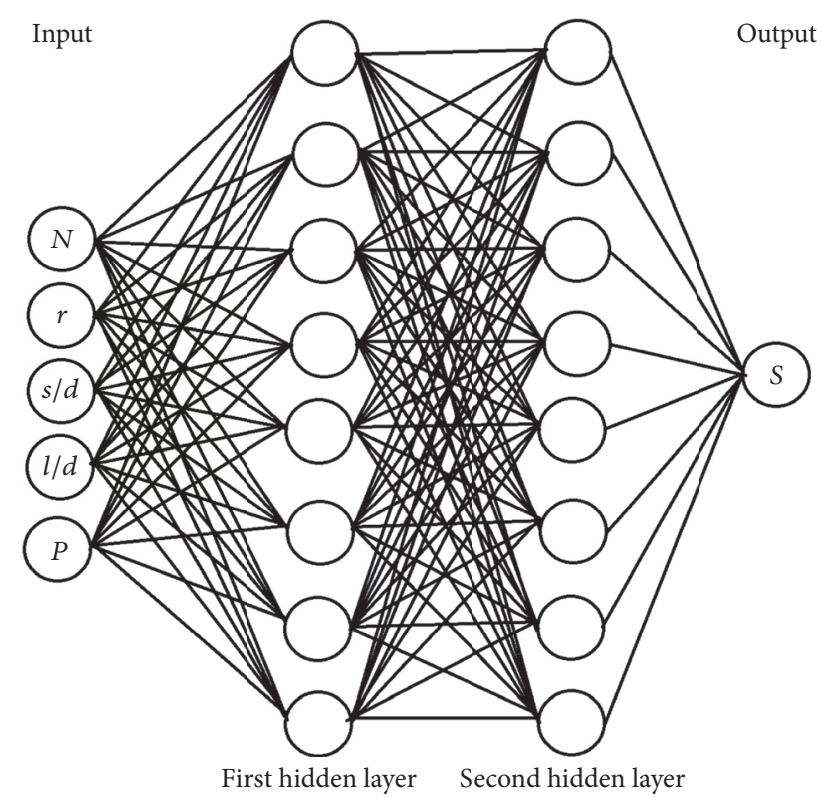

FIGURE 15: Multilayer perceptron neural network.

squares method to estimate the constants of a previously evolutionary developed model [40]. The general steps applied in EPR are depicted in Figure 17.

In this method, type of adopted functions, number of terms exponents' range, and the number of generations are the parameters which affect the output relationship [31]. Besides, similar to the neural network modeling, the coefficient of determination evaluates the degree of accuracy of

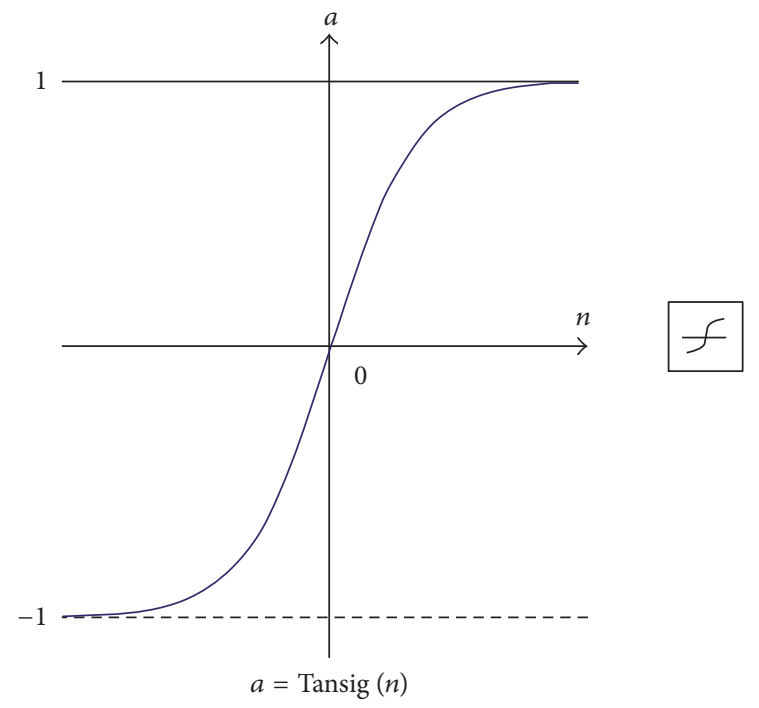

FIGURE 16: Nonlinear TANSIG transfer function [34].

the proposed equation. If the proposed model satisfied both termination criteria (maximum number of generations and sentences) and required prediction accuracy, it will terminate. Otherwise, it goes through another evolution [32].

\section{Results}

In order to evaluate and compare the developed models and techniques, their performances have been investigated in different various terms. 


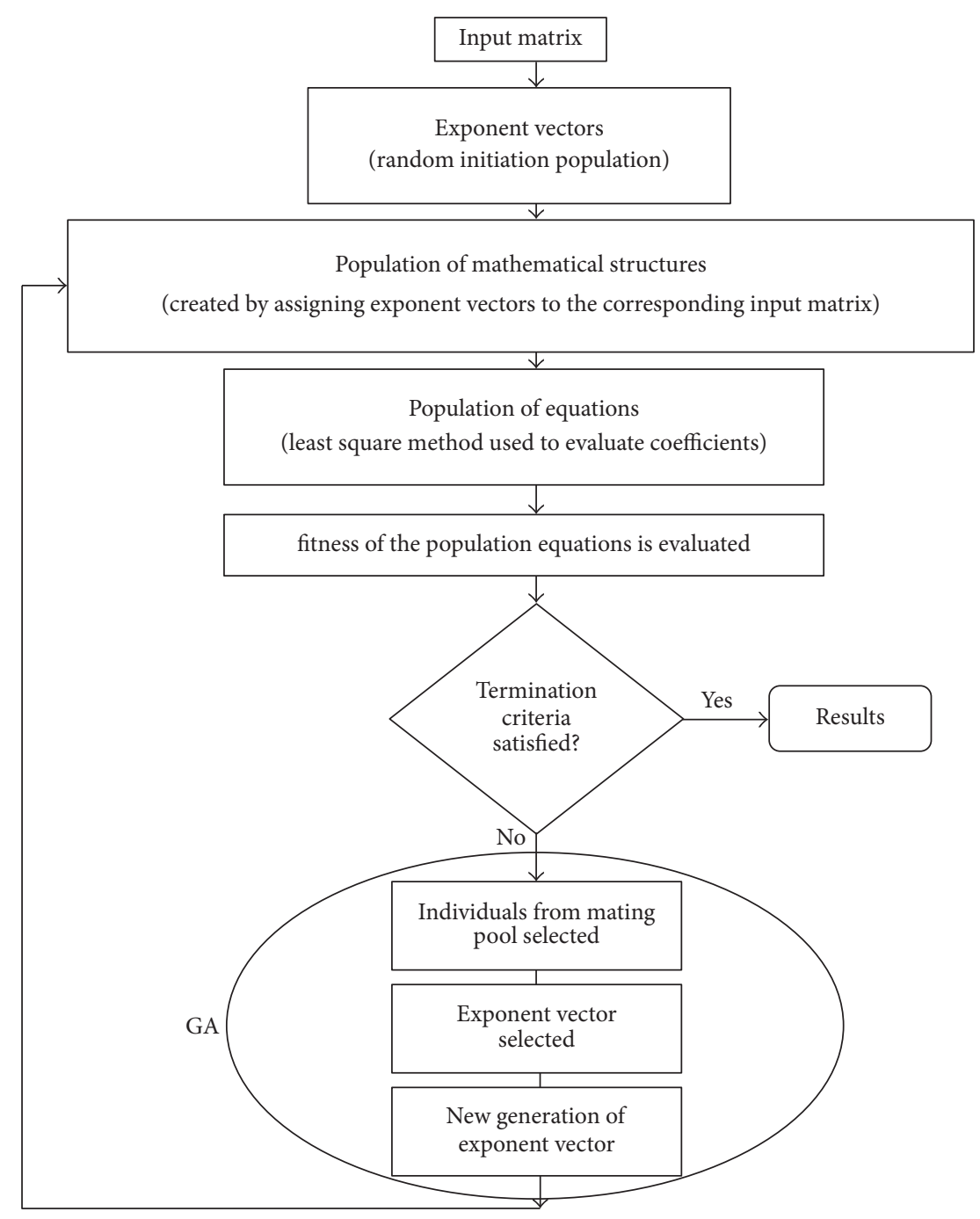

FIgURE 17: Typical flow diagram for EPR procedure [35].

6.1. Neural Network Modeling of Static and Coupled StaticDynamic Loading Induced Settlement of CPRF

6.1.1. Architectures of the Best Neural Networks. Different oneand two-layered networks with different neurons for each hidden layer were built and trained. In order to evaluate the constructed networks, the value of root mean squared error, RMSE, and the coefficient of determination, $R^{2}$, were calculated. For all the built models, the value of root mean squared error, RMSE, and mean absolute error and, also, coefficient of correlation, $R^{2}$, were calculated and compared. The applied formula for calculating RMSE is presented in [29]

$$
\mathrm{RMSE}=\sqrt{\frac{\sum\left(O_{i}-T_{i}\right)^{2}}{n}},
$$

where $T_{i}$ and $O_{i}$ are calculated and forecasted outputs, respectively. Also, $n$ is the number of datasets.
6.1.2. Neural Network Performances. To evaluate the performance of the optimum models, the normalized predicted values of static and combined static-dynamic settlements (for testing data series) are predicted using both conventional and genetic algorithm based neural networks.

(1) Performance of Neural Networks in the Static Loading Case. The value of RMSE and coefficient of correlation along with the slope of fitting line, $A$, for different network architectures are shown in Table 3, where different terms, digits, of each of the network architectures represent the number of neurons in its corresponding layers.

Figure 18 presents correlation between predicted settlements versus calculated ones for the best network, 5-3-12-1.

Moreover, the predicted and calculated datasets are shown in Figure 19.

(2) Performance of Neural Networks in the Combined StaticDynamic Loading Case. The results of evaluations of the 
TABLE 3: Performance of neural networks in the static case.

\begin{tabular}{lccr}
\hline Network architecture & RMSE & $A$ & $R^{2}$ \\
\hline $5-5-1$ & 0.2379 & -0.025 & 0.153 \\
$5-10-1$ & 0.2371 & 0.032 & 0.173 \\
$5-13-1$ & 0.1275 & 0.024 & 0.131 \\
$\mathbf{5 - 3 - 1 2 - 1}$ & $\mathbf{0 . 1 8 2 9}$ & $\mathbf{0 . 9 6 7}$ & $\mathbf{0 . 8 9}$ \\
$5-5-10-1$ & 0.2075 & -0.006 & 0.221 \\
\hline
\end{tabular}

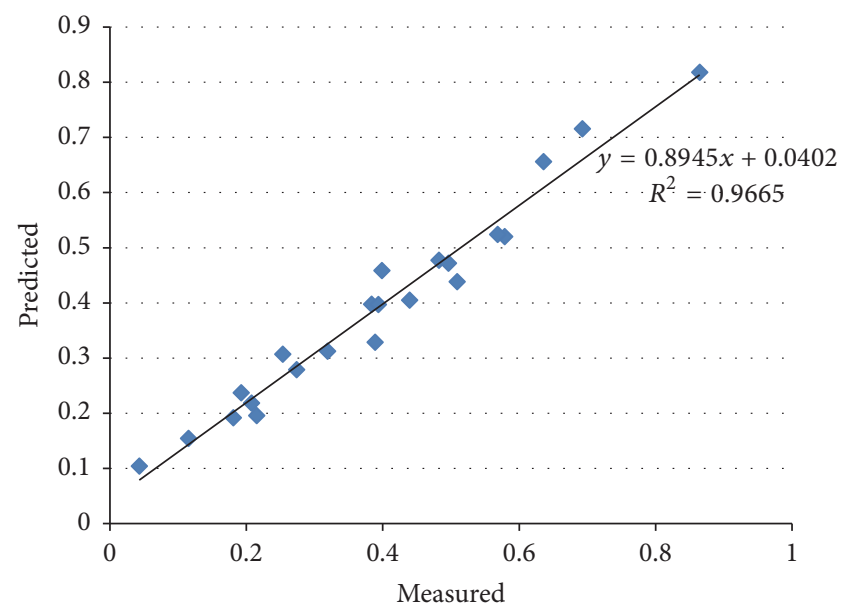

FIGURE 18: Correlation between predicted settlements versus calculated ones for the best static neural network, 5-3-12-1.

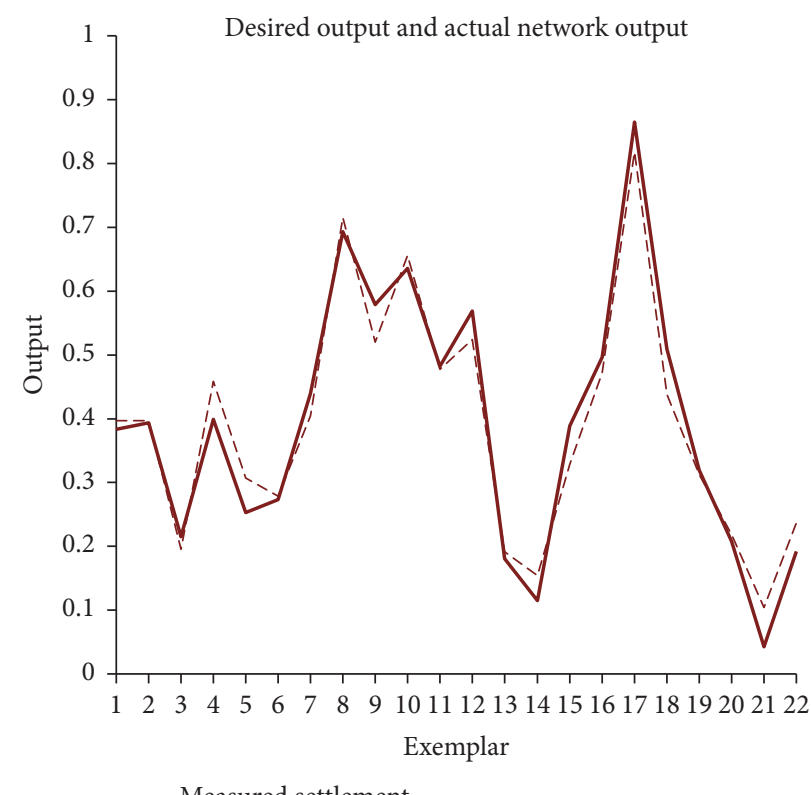

- - Predicted settlement

FIGURE 19: Predicted settlements versus calculated ones for the best static neural network, 5-3-12-1.

developed models in the coupled static-dynamic loading cases are presented in Table 4 .

Figure 20 presents correlation between predicted settlements versus calculated ones for the best network, 5-8-8-1.

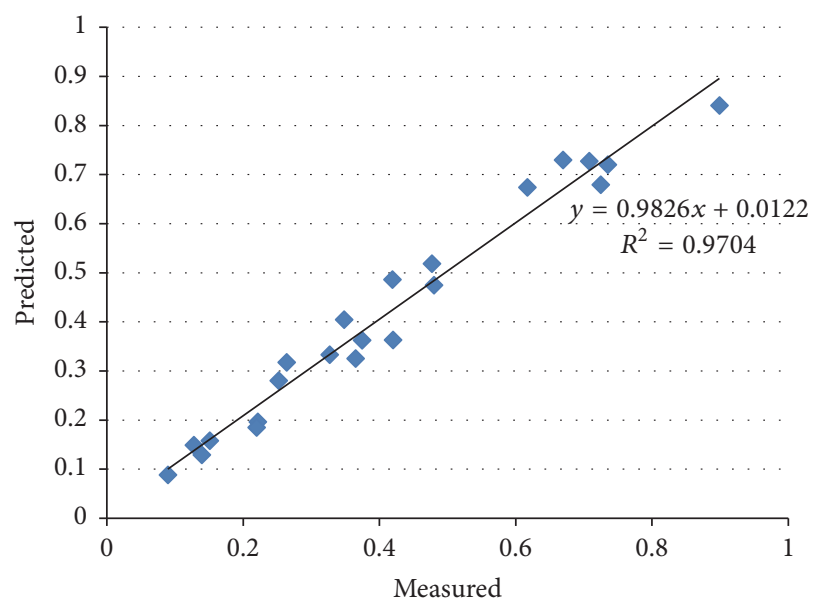

FIGURE 20: Correlation between predicted settlements versus calculated ones for the best static- dynamic neural network, 5-8-8-1.

Moreover, the predicted and calculated datasets are shown in Figure 21.

6.2. EPR Models. Using EPR modeling, different relations have been developed for both static and coupled staticdynamic settlements based on input parameters. Equations (10) show the best optimum relations for settlement prediction in static and coupled static-dynamic loading conditions, respectively.

$$
\begin{aligned}
S_{s}= & -0.46228\left(p^{0.5}\right) \ln \left[\left(\frac{l}{d}\right)+1\right]^{0.5} \ln (p+1) \\
& +0.1153(p) \ln \left[\left(\frac{s}{d}\right)+1\right]^{0.5} \\
& +0.0034446\left(p^{2}\right)+0.12898\left(N_{p}\right)\left(d^{0.5}\right) \\
& +26.5835,
\end{aligned}
$$

$$
\begin{aligned}
S_{\text {csd }}= & 0.16409(p)\left(\frac{l}{d}\right) \operatorname{sech}\left(\frac{l}{d}\right)^{0.5} \\
& +9.1863\left(d^{0.5}\right)\left(p^{0.5}\right) \operatorname{sech}\left(\frac{s}{d}\right) \\
& +0.97359 N_{p}^{0.5}+1.7638,
\end{aligned}
$$


TABLE 4: Performance of neural networks in the coupled static-dynamic loading case.

\begin{tabular}{lccc}
\hline Network architecture & RMSE & $A$ & $R^{2}$ \\
\hline $5-5-1$ & 0.2508 & 0.016 & 0.271 \\
$5-10-1$ & 0.298 & -0.016 & 0.251 \\
$5-13-1$ & 0.2441 & 0.026 & 0.22 \\
$5-3-12-1$ & 0.3391 & -0.001 & 0.258 \\
$5-5-10-1$ & 0.3115 & -0.004 & 0.824 \\
$\mathbf{5 - 8 - 8 - 1}$ & $\mathbf{0 . 0 3 9}$ & $\mathbf{0 . 9 8 3}$ & $\mathbf{0 . 9 7}$ \\
\hline
\end{tabular}

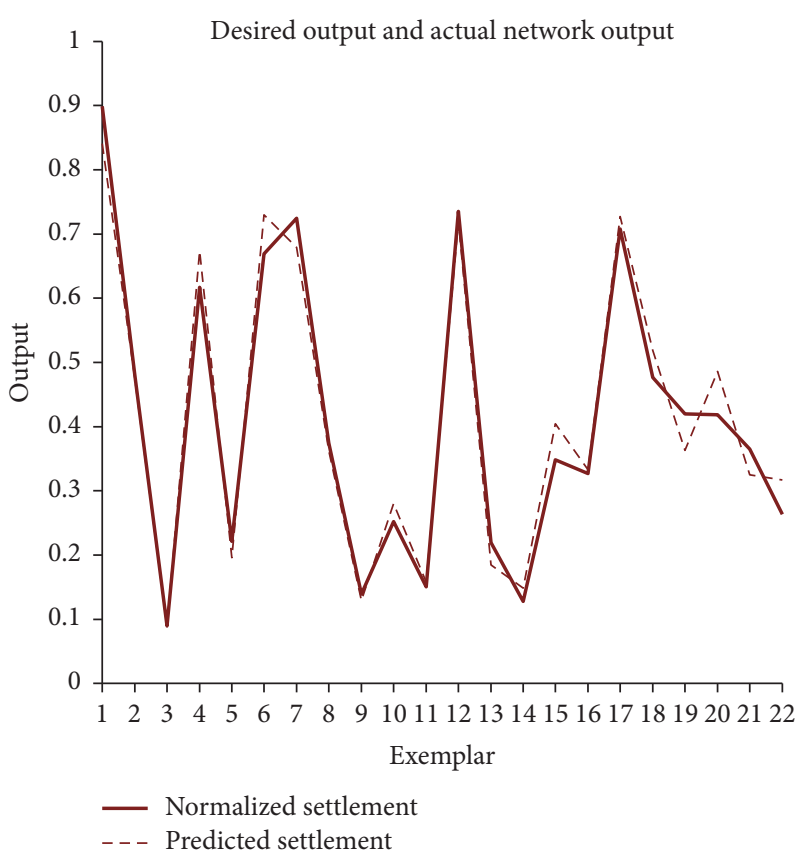

FIGURE 21: Predicted settlements versus calculated ones for the best static-dynamic neural network, 5-8-8-1.

where $S_{s}, S_{\mathrm{csd}}, p, l, d, s$, and $N_{p}$, respectively, represent static settlement, coupled static-dynamic settlement, axial static load, pile length, pile diameter, spacing, pile radius, and number of piles. Also, $\operatorname{sech}(x)$ is the hyperbolic secant of $(x)$.

As it is shown in Figures 22 and 23, the coefficients of determination for both static and static-dynamic coupled models presented using EPR are acceptable values of $97.49 \%$ and $97.26 \%$, respectively, which mean that the proposed models are capable for predicting the settlements based on the studied input parameters. Moreover, the values of RMSE for static and coupled static-dynamic settlement models are 0.271 and 0.364 , respectively.

6.3. Sensitivity Analysis. The strength of relationship between affecting parameters (number of piles, radius, ratio of $s / d$, ratio of $l / d$, and axial force) and the values of settlements of CPRF in both static and coupled static-dynamic conditions can be found using sensitivity analysis based on Cosine Amplitude Method (CAM) [33] of CPRF in both static and coupled static-dynamic conditions.

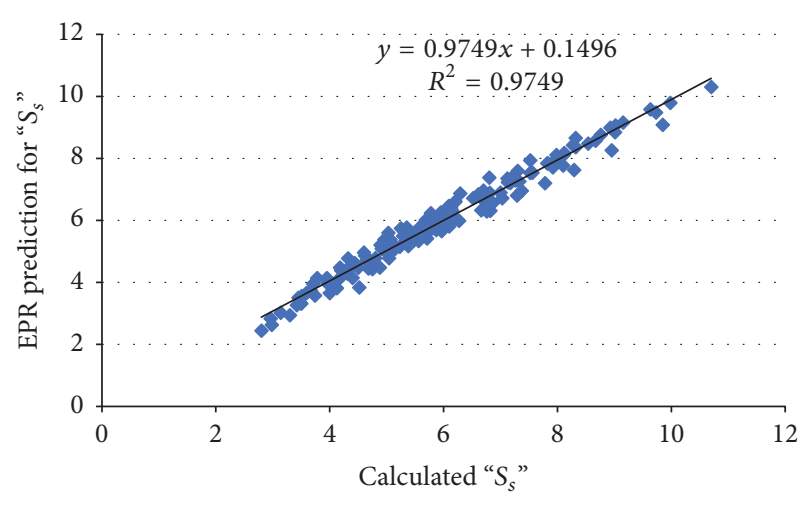

FIGURE 22: The calculated values of static settlement versus its corresponding EPR prediction.

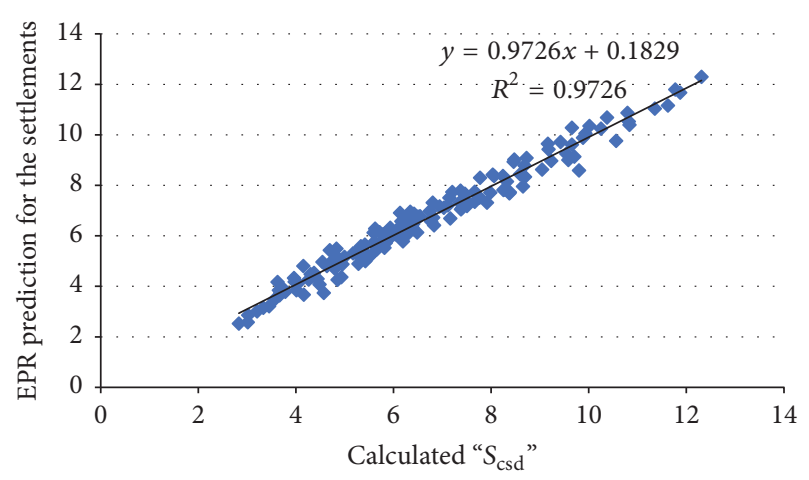

FIGURE 23: The calculated values of coupled static-dynamic settlements versus its corresponding EPR prediction.

In this method, the strength of relationship between target parameter and inputs is found using [33, 41]:

$$
r_{i j}=\frac{\sum_{m=1}^{k} x_{i m} x_{j m}}{\sqrt{\sum_{m=1}^{k} x_{i m}^{2} \sum_{m=1}^{k} x_{j m}^{2}}}
$$

where each of the input parameters is expressed as one of $X$ array's elements shown in [33]

$$
X=\left\{x_{1}, x_{1}, \ldots, x_{i}, \ldots, x_{n}\right\}
$$




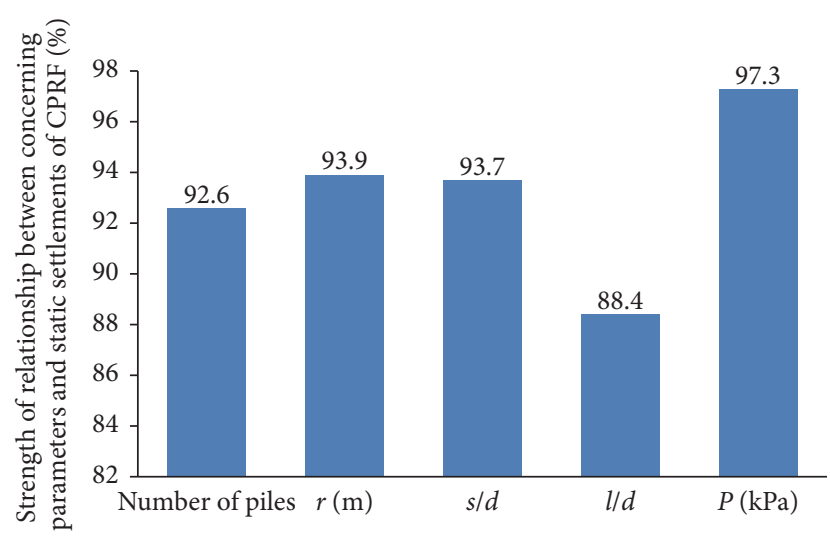

FIGURE 24: Strength of relationship between input parameters and CPRF settlements in the static case.

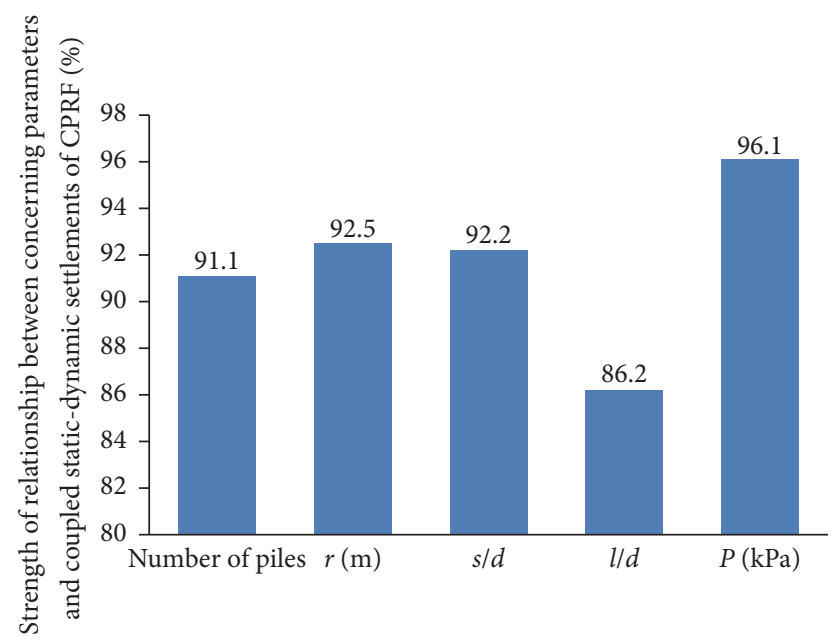

FIGURE 25: Strength of relationship between input parameters and CPRF settlements in the coupled static-dynamic case.

where each of its elements is a vector with the length of $k$ and is presented in [33]

$$
x_{i}=\left\{x_{i 1}, x_{i 2}, x_{i 3}, \ldots x_{i k}\right\} .
$$

Figures 24 and 25 show the strength of relationship between input parameters and CPRF settlements in static and coupled static-dynamic case, respectively. As it can be seen, axial load is the most important parameter in both conditions. Also, $l / d$ is the least effective parameter on the static and coupled static-dynamic CPRF's settlements. Besides, effects of other concerning parameters on the settlements are quiet the same.

\section{Conclusion}

Knowing the piled raft settlements in static and coupled static-dynamic loading conditions plays an important role in preliminary design phase of these combined foundations. Regarding the high expenses of full-scale model tests and experimental tests in determining the earthquake induced settlements of the combined piled raft foundation and, also, crucial need for quite a speedy and inexpensive predictive method, the applicability of neural network modeling has been investigated and compared. Moreover, the large calculation times available in commercial finite element or finite difference software programs for dynamic complicated problems makes the problem time-consuming. Hence, a speedy and accurate tool for predicting these values will significantly help the engineers to facilitate the design procedure. In this regard, the highly in-demand predictive tools, ANN, and EPR modeling have been considered to forecast the settlements in both static and coupled dynamic-static loading conditions. In this regard, dynamic nonlinear finite difference modeling has been used to prepare the required datasets. After wellvalidating the used numerical procedure and applying a parametric study, a databank consisting of 144 datasets has been gained. Using the developed databank, different ANNs and polynomial regression models have been constructed, which can be efficiently used to predict the values of settlements for all the cases that there are similar available input parameters. Moreover, using the sensitivity analysis, the most and the least effective parameters on the static and coupled static-dynamic settlements have been determined. Based on the obtained results, the following can be concluded:

(i) Neural networks are introduced as capable effective tools for predicting static and coupled static-dynamic settlements of CPRFs.

(ii) The best neural network in static case is 5-3-12-1 FFBP neural network with RMSE of $0.1829, R^{2}$ of 0.967 , and $A$ of 0.89 .

(iii) In the case of coupled static-dynamic loads, the network with the architecture of 5-8-8-1, RMSE of $0.039, R^{2}$ of 0.97 , and $A$ of 0.983 is the best obtained neural network.

(iv) Based on EPR modeling, a new relationship with coefficient of determination of $97.49 \%$ and RMSE of 0.271 and the line slope of 0.975 for predicting the static settlements of combined piled raft foundations has been proposed.

(v) A new relationship with coefficient of determination of $97.26 \%$ and RMSE of 0.137 and the line slope of 0.973 for predicting the coupled static-dynamic settlements of combined piled raft foundations has been presented.

(vi) Sensitivity analysis emphasized that axial load plays an important role in calculating static/coupled staticdynamic settlements and neglecting its effects will lead to significant errors in calculations. It was also shown that the effect of $l / d$ on the settlements is less than other concerning parameters.

Based on the results, some efficient and highly capable tools for predicting static and coupled static-dynamic settlements of CPRFs are suggested, which can be successfully used to predict the values of settlements in preliminary step of CPRF design. 


\section{Conflicts of Interest}

The authors declare that they have no conflicts of interest.

\section{References}

[1] R. W. Cooke, "Piled raft foundations on stiff clays - a contribution to design Philosophy," Geotechnique, vol. 36, no. 2, pp. 169-203, 1986.

[2] J. B. Burland, "Piles as settlement reducer," in Proceedings of 18th Italian Congress on Soil Mech, Pavia, 1995.

[3] H. G. Poulos, "Piled raft foundations: design and applications," Geotechnique, vol. 51, no. 2, pp. 111-113, 2001.

[4] R. Katzenbach, V. Arslan, and C. Moorman, "Numerical stimulations of combined piled raft foundations for the new high rise building. Max in Frankfurt am main," in Proceedings of the 2nd International Conference on Soil Structure Interaction in Urban Civil Engineering, ETH, Zurich, Switzerland, March 2000.

[5] V. Balakumar and K. Ilamparuthi, "Performance monitoring of a piled raft foundation of twelve storied building and analytical validation," Indian geotechnical Journal, vol. 37, no. 2, pp. 94-115, 2007.

[6] M. H. Baziar, A. Ghorbani, and R. Katzenbach, "Small-scale model test and three-dimensional analysis of pile-raft foundation on medium-dense sand," International Journal of Civil Engineering, vol. 7, no. 3, pp. 170-175, 2009.

[7] K. Horikoshi, Optimum Design of Piled Raft Foundations. Dissertation for the degree of Doctor of Philosophy [Dissertation, thesis], University of Western Australia, 1995.

[8] V. Balakumar, V. Kalaiarasi, and K. Ilamparuthi, "Experimental and analytical study on the behaviour of circular piled raft on sand," in Proceedings of the 16th International Conference on Soil Mechanics and Geotechnical Engineering, Osaka, Japan, 2005.

[9] R. Saha, S. C. Dutta, and S. Haldar, "Seismic response of soilpile raft-structure system," Journal of Civil Engineering and Management, vol. 21, no. 2, pp. 144-164, 2015.

[10] J. X. Lai, H. Q. Liu, J. L. Qiu, and J. Chen, "Settlement analysis of saturated tailings dam treated by CFG pile composite foundation," Advances in Materials Science and Engineering, vol. 2016, Article ID 7383762, 10 pages, 2016.

[11] A. Kumar, D. Choudhury, and R. Katzenbach, "Effect of earthquake on combined pile-raft foundation," International Journal of Geomechanics, vol. 16, no. 5, Article ID 04016013, 2016.

[12] H. G. Polous, "The piled raft foundation for the Burj Dubai," Design and Performance, 2008.

[13] K. Yamashita, J. Hamada, and T. Tanikawa, "Field measurements on piled rafts with grid-form deep mixing walls on soft ground," Geaotechnical Engineering-SEAGS, vol. 42, no. 2, 2010.

[14] D. J. Armaghani, R. S. N. S. B. R. Shoib, K. Faizi, and A. S. A. Rashid, "Developing a hybrid PSO-ANN model for estimating the ultimate bearing capacity of rock-socketed piles," Neural Computing and Applications, vol. 28, no. 2, pp. 391-405, 2017.

[15] M. H. Baziar, A. S. Azizkandi, and A. Kashkooli, "Prediction of pile settlement based on cone penetration test results: an ann approach," KSCE Journal of Civil Engineering, vol. 19, no. 1, pp. 98-106, 2014.

[16] A. Ghorbani and H. Hasanzadehshooiili, "A novel solution for ground reaction curve of tunnels in elastoplastic strain softening rock masses," Journal of Civil Engineering and Management, vol. 23, no. 6, pp. 773-786, 2017.
[17] R. A. A. Heshmati, A. H. Alavi, M. Keramati, and A. H. Gandomi, "A radial basis function neural network approach for compressive strength prediction of stabilized soil," GeoHunan International Conference, pp. 147-153, 2009.

[18] U. Dikmen and M. Sonmez, "An artificial neural networks model for the estimation of formwork labour," Journal of Civil Engineering and Management, vol. 17, no. 3, pp. 340-347, 2011.

[19] A. Ghorbani, Y. Jafarian, and M. S. Maghsoudi, "Estimating shear wave velocity of soil deposits using polynomial neural networks: application to liquefaction," Computers and Geosciences, vol. 44, pp. 86-94, 2012.

[20] Ł. Sadowski and M. Nikoo, "Corrosion current density prediction in reinforced concrete by imperialist competitive algorithm," Neural Computing and Applications, vol. 25, no. 7-8, pp. 1627-1638, 2014.

[21] Ł. Sadowski, J. Hoła, and S. Czarnecki, "Non-destructive neural identification of the bond between concrete layers in existing elements," Construction and Building Materials, vol. 127, pp. 49$58,2016$.

[22] Ł. Sadowski, "Non-destructive identification of pull-off adhesion between concrete layers," Automation in Construction, vol. 57, pp. 146-155, 2015.

[23] M. H. Baziar and A. Ghorbani, "Evaluation of lateral spreading using artificial neural networks," Soil Dynamics and Earthquake Engineering, vol. 25, no. 1, pp. 1-9, 2005.

[24] S. S. H. Yasrebi and M. Emami, "Application of artificial neural networks (ANNs) in prediction and interpretation of pressuremeter test results," in Proceeding of the 12th International Conference of International Association for Computer Methods and Advances in Geomechanics (IACMAG '08), Goa, India, 2008.

[25] M. Monjezi, A. Bahrami, and A. Yazdian Varjani, "Simultaneous prediction of fragmentation and flyrock in blasting operation using artificial neural networks," International Journal of Rock Mechanics and Mining Sciences, vol. 47, no. 3, pp. 476-480, 2010.

[26] K. M. Neaupane and N. R. Adhikari, "Prediction of tunnelinginduced ground movement with the multi-layer perceptron," Tunnelling and Underground Space Technology, vol. 21, no. 2, pp. 151-159, 2006.

[27] Y. Yang and M. S. Rosenbaum, "The artificial neural network as a tool for assessing geotechnical properties," Geotechnical and Geological Engineering, vol. 20, no. 2, pp. 149-168, 2002.

[28] H. Hasanzadehshooiili, A. Lakirouhani, and J. Medzvieckas, "Superiority of artificial neural networks over statistical methods in prediction of the optimal length of rock bolts," Journal of Civil Engineering and Management, vol. 18, no. 5, pp. 655-661, 2012.

[29] H. Hasanzadehshooiili, R. Mahinroosta, A. Lakirouhani, and V. Oshtaghi, "Using artificial neural network (ANN) in prediction of collapse settlements of sandy gravels," Arabian Journal of Geosciences, vol. 7, no. 6, pp. 2303-2314, 2014.

[30] F. Meulenkamp and M. A. Grima, "Application of neural networks for the prediction of the unconfined compressive strength from Equotip hardness," International Journal of Rock Mechanics and Mining Sciences, vol. 36, no. 1, pp. 29-39, 1999.

[31] M. Rezania, A. A. Javadi, and O. Giustolisi, "An evolutionarybased data mining technique for assessment of civil engineering systems," Engineering Computations, vol. 25, no. 6, pp. 500-517, 2008. 
[32] A. Ahangar-Asr, A. Faramarzi, N. Mottaghifard, and A. A. Javadi, "Modeling of permeability and compaction characteristics of soils using evolutionary polynomial regression," Computers and Geosciences, vol. 37, no. 11, pp. 1860-1869, 2011.

[33] A. Ghorbani, H. Hasanzadehshooiili, E. Ghamari, and J. Medzvieckas, "Comprehensive three dimensional finite element analysis, parametric study and sensitivity analysis on the seismic performance of soil-micropile-superstructure interaction," Soil Dynamics and Earthquake Engineering, vol. 58, pp. 21-36, 2014.

[34] FLAC3D, Fast Lagrangian Analysis of Continua in 3 Dimensions, Version 3.1. Itasca: Minneapolis. Itasca Consulting Group, Inc.

[35] P. K. Simpson, Artificial Neural System: Foundation, Paradigms, Applications and Implementations, Pergamon Press, New York, 1990.

[36] T. Belytschko, "An overview of semi discretization and time integration procedures," in Computational Methods for Transient Analysis. Computational Methods in Mechanics Series. 1. North, T. Belytschko and T. J. R. Hughes, Eds., NY, USA, 1983.

[37] K.-I. Funahashi, "On the approximate realization of continuous mappings by neural networks," Neural Networks, vol. 2, no. 3, pp. 183-192, 1989.

[38] W. S. Sarle, "Neural Networks and Statistical Models," in Proceeding ofof the Nineteenth Annual SAS Users Group International Conference, 1994.

[39] H. Demuth, M. Beal, and M. Hagan, Neural network toolbox 5 users guide. The Math Work, 1996.

[40] O. Giustolisi and D. A. Savic, "A symbolic data-driven technique based on evolutionary polynomial regression," Journal of Hydroinformatics, vol. 8, no. 3, pp. 207-222, 2006.

[41] A. Ghorbani, H. Hasanzadehshooiili, A. Šapalas, and A. Lakirouhani, "Buckling of the steel liners of underground road structures: The sensitivity analysis of geometrical parameters," The Baltic Journal of Road and Bridge Engineering, vol. 8, no. 4, pp. 250-254, 2013. 

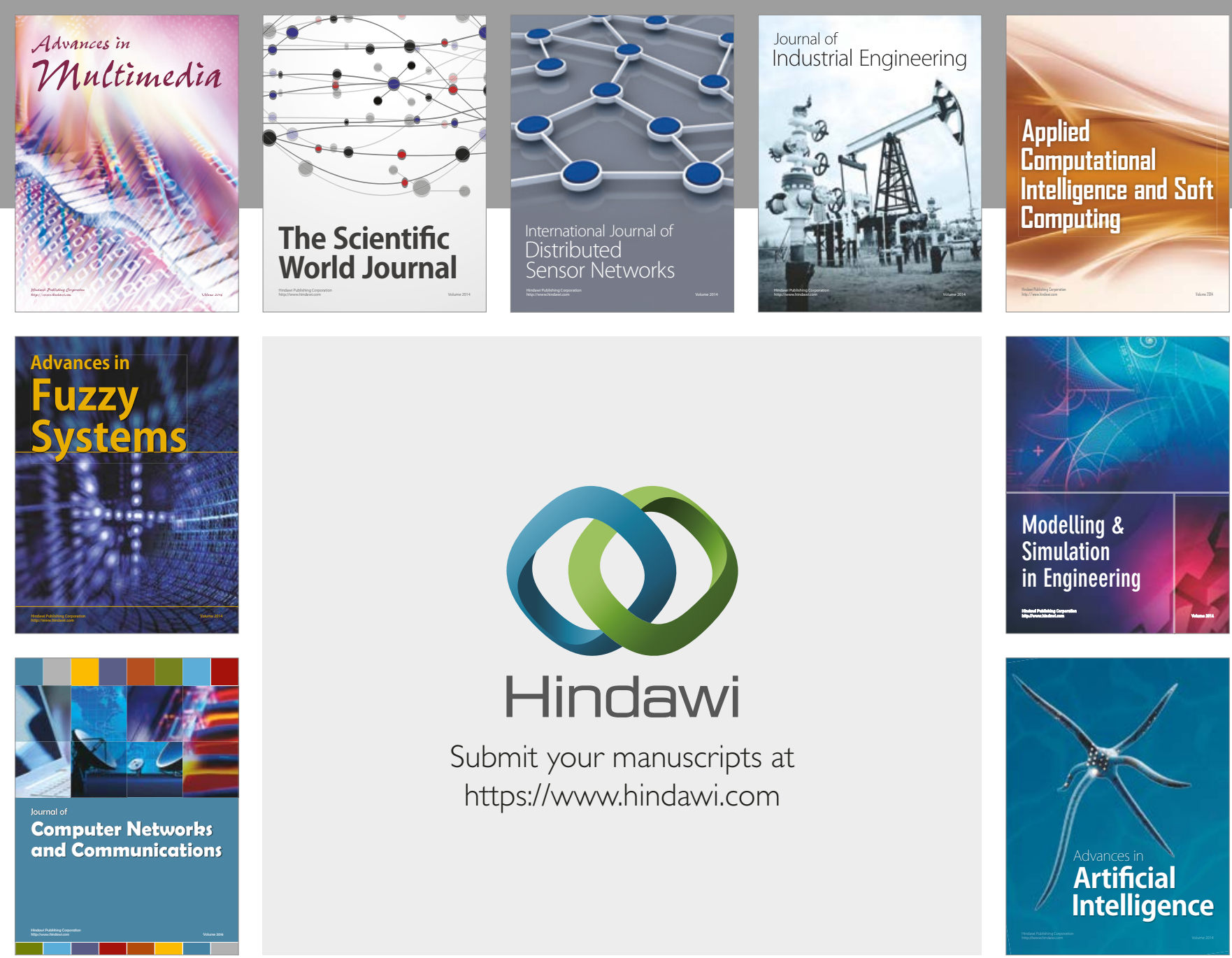

\section{Hindawi}

Submit your manuscripts at

https://www.hindawi.com
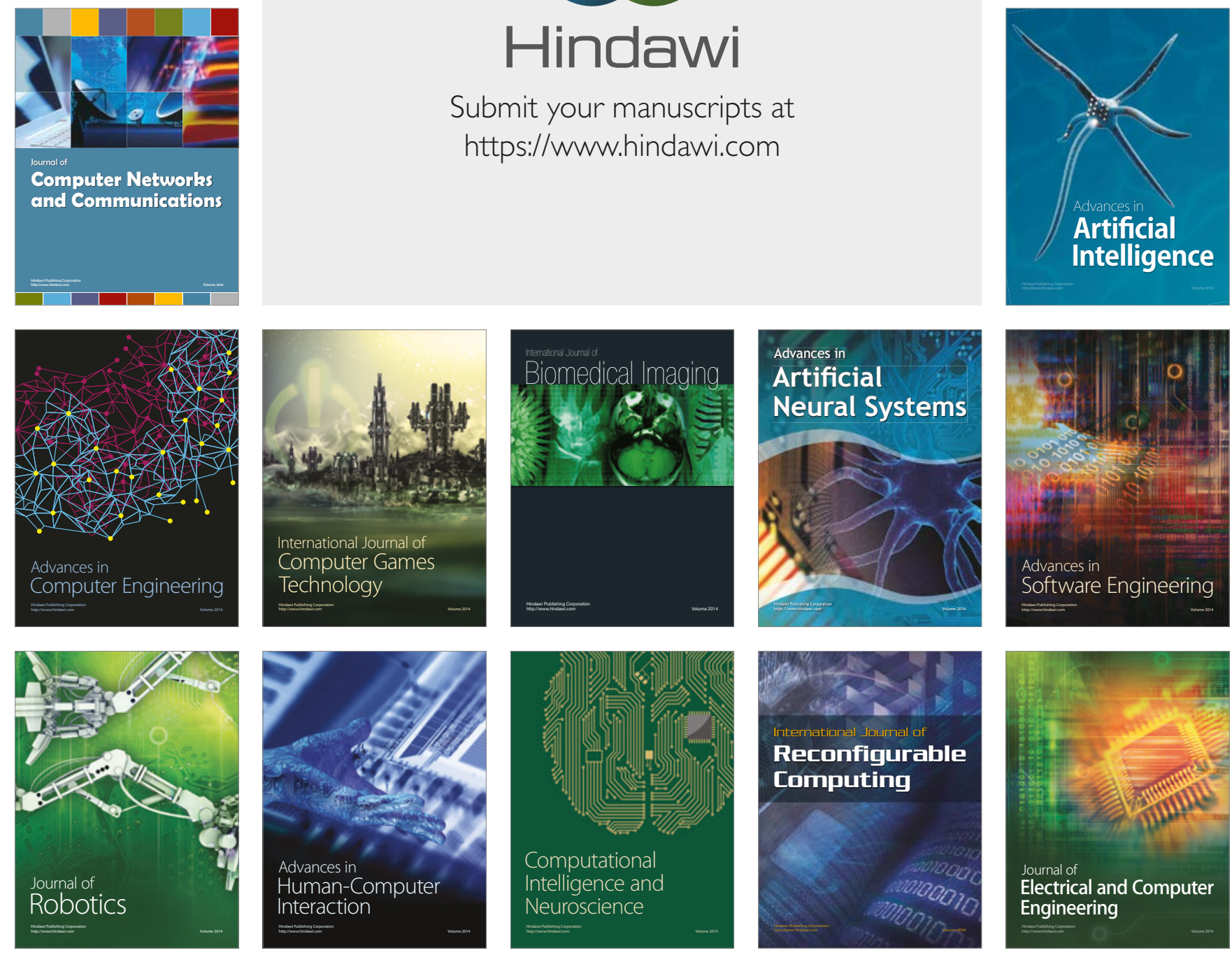